\title{
ANALYTICAL BOUNDS \\ FOR TWO VALUE-AT-RISK FUNCTIONALS
}

BY

\author{
WERNER HÜRLIMANN
}

\begin{abstract}
Based on the notions of value-at-risk and conditional value-at-risk, we consider two functionals, abbreviated $\mathrm{VaR}$ and $\mathrm{CVaR}$, which represent the economic risk capital required to operate a risky business over some time period when only a small probability of loss is tolerated. These functionals are consistent with the risk preferences of profit-seeking (and risk averse) decision makers and preserve the stochastic dominance order (and the stop-loss order). This result is used to bound the VaR and CVaR functionals by determining their maximal values over the set of all loss and profit functions with fixed first few moments. The evaluation of CVaR for the aggregate loss of portfolios is also discussed. The results of VaR and CVaR calculations are illustrated and compared at some typical situations of general interest.
\end{abstract}

\section{KEYWORDS}

Value-at-risk, Conditional value-at-risk, Economic risk capital, Ordering of risks, Stochastic bounds, Comonotonic risks

\section{INTRODUCTION AND PRELIMINARIES}

The present contribution shows how some elementary principles and methods in measurement of risk can be used to calculate appropriate amounts of risk capital required to operate a risky business over some time period. The emphasis is on distribution-free methods, which require for a practical implementation only the knowledge of the first few moments, namely the mean, variance, skewness and kurtosis. The interest for such methods is widespread and still actual (e.g. Li (1999)). First, a brief overview of the content follows. Then, we introduce a minimal number of important notions, definitions and results, which will be used throughout.

Based on the notions of value-at-risk and conditional value-at-risk, we introduce in Section 2 two functionals, abbreviated VaR and CVaR, which represent the economic risk capital required to operate a risky business over some time period when only a small probability of loss is tolerated. It is recalled 
that VaR (resp. CVaR) is consistent with the risk preferences of profit-seeking (resp. profit-seeking risk averse) decision makers. Both functionals preserve the corresponding ordering of risks, namely the stochastic dominance order (resp. the stop-loss order). When only incomplete information about the loss and profit function of a risky business is available, then this order preserving result is used to bound the VaR and CVaR functionals by determining their extremal values over the set of all loss and profit functions with fixed first few moments. This procedure is useful in practice, especially if one agrees to compute capital requirements, not on the actual loss and profit function, but on a stochastic upper bound for this loss in its moment space.

The maximum of $\mathrm{VaR}$ and $\mathrm{CVaR}$ by known range, mean and variance is obtained in Theorem 3.1. In particular, it is shown that if the loss tolerance level is sufficiently small, then these maximal values coincide. As an illustration, we determine in Example 3.1 the minimum risk capital required by a company whose loss and profit function is known to belong to a finite range with known mean $\mu$ and coefficient of variation $k$. It equals $\left(1+k^{2}\right) \cdot \mu$ and is related to several other quantities of interest, namely the Karlsruhe price by Heilmann (1987), the limiting CAPM fair premium in Hürlimann(1994), Theorem 4.1, and the Hardy-Littlewood price for large $k$ introduced in Hürlimann(1998a).

Similarly, the maximum of VaR and CVaR by known mean, variance, skewness, kurtosis and range $(-\infty, \infty)$ is determined in Section 4 . Corollary 4.1 shows that if the loss tolerance level is sufficiently small, then the maximum of the VaR and CVaR functionals coincide and identify with a simple analytical expression.

The evaluation of the maximum VaR and CVaR for the aggregate loss of portfolios is discussed in Section 5. It is recalled that the maximum CVaR for the aggregate loss of a portfolio with fixed marginal losses is attained at the portfolio with mutually comonotonic components, and that if the marginal losses have absolutely continuous distributions, then the maximum $\mathrm{CVaR}$ is an additive functional. This result yields in Theorem 5.1 a simple recipe for the calculation of the maximum CVaR for the aggregate loss of portfolios given incomplete information about the marginal losses.

Section 6 illustrates and compares results of $\mathrm{VaR}$ and $\mathrm{CVaR}$ calculations at some typical situations of general interest. Table 6.1 compares VaR and CVaR for two-parameter distributions with fixed mean and variance by varying loss probability. Several anomalies are noted and suggest that a capital requirement based on the right-tail risk measure of Wang(1998), extensively studied in Hürlimann(2000), is worth to be considered in future work. Table 6.2 compares $\mathrm{VaR}, \mathrm{CVaR}$ and the maximum $\mathrm{CVaR}$ for a log-normal distribution by varying parameters but fixed tolerance probability. Compared to the obtained exact value, the maximum $\mathrm{CVaR}$ contains only a small implicit margin. Timedependent comparisons are made in Table 6.3 while Table 6.4 displays the dependence upon the kurtosis parameter for two important symmetric distributions. Finally, Table 6.5 discusses the capital requirements of a classical insurance risk model.

Capital letters $X, Y, \ldots$ denote random variables with distribution functions $F_{X}(x), F_{Y}(x)$, and finite means $\mu_{X}, \mu_{Y}$. The survival function of $X$ is denoted 
by $\bar{F}_{X}(x)=1-F_{X}(x)$. The stop-loss transform of a random variable $X$ is defined by

$$
\pi_{x}(x):=E\left[(X-x)_{+}\right]=\int_{x}^{\infty} \bar{F}_{X}(t) d t, x \text { in the support of } X .
$$

The random variable $X$ is said to precede $Y$ in stochastic order or stochastic dominance of first order, a relation written as $X \leq_{s t} Y$, if $\bar{F}_{X}(x) \leq \bar{F}_{Y}(x)$ for all $x$ in the common support of $X$ and $Y$. The random variables $X$ and $Y$ satisfy the stop-loss order, or equivalently the increasing convex order, written as $X \leq_{s l} Y$ (or $X \leq_{i c x} Y$ ), if $\pi_{X}(x) \leq \pi_{Y}(x)$ for all $x$. The attractiveness of the partial order relations $\leq_{s t}$ and $\leq_{s l}$ is corroborated by several invariance properties (e.g. Kaas et al.(1994), chap. II.2 and III.2, or Shaked and Shanthikumar(1994)). For example, both of $\leq_{s t}$ and $\leq_{s l}$ are closed under convolution and compounding, and $\leq_{s l}$ is additionally closed under mixing and conditional compound Poisson summing.

Given a partial order between random variables and some class of random variables, it is possible to construct extremal random variables with respect to this partial order, which provide useful information about extreme situations in probabilistic modelling (e.g. Stoyan(1977)). For example, the classical ChebyshevMarkov probability inequalities yield the extremal random variables with respect to the usual stochastic order for the class of random variables with a given range and moments known up to a fixed number. Extremal random variables with respect to the stop-loss order are of similar general interest. The extremal random variables with respect to these main stochastic orders find important actuarial and financial applications (e.g. Hürlimann(1996,1997a/b,1998a/b, 1999,2001a).

The class of all random variables with given support, $[A, B],-\infty \leq A<B$ $\leq \infty$, and known moments $\mu_{1}, \mu_{2}, \ldots, \mu_{n}$ is denoted by $D_{n}\left([A, B] ; \mu_{1}, \mu_{2}, \ldots, \mu_{n}\right)$ or simply $D_{n}$ in case the context is clear. For each fixed $n=2,3,4, \ldots$, we denote by $F_{s t, \max }^{(n)}(x), F_{s, \text { min }}^{(n)}(x)$ the Chebyshev-Markov extremal distributions, which are solutions of the extremal moment problems

$$
F_{s \hbar \max }^{(n)}(x)=\min _{X \in D_{n}}\left\{F_{X}(x)\right\}, F_{s \zeta \text { min }}^{(n)}(x)=\max _{X \in D_{n}}\left\{F_{X}(x)\right\} .
$$

Random variables with distributions $F_{s, \text { max }}^{(n)}(x), F_{s t, \min }^{(n)}(x)$ are denoted by $X_{s t, \max }^{(n)}$, $X_{s t, \min }^{(n)}$, and are extremal with respect to the usual stochastic order, that is one has $X_{s t, \min }^{(n)} \leq_{s t} X \leq_{s t} X_{s t, \max }^{(n)}$ for all $X \in D_{n}$. For fixed $n=2,3,4$, the minimal and maximal stop-loss transforms over the space $D_{n}$, which are defined and denoted by $\pi_{\min }^{(n)}(x):=\min _{X \in D_{n}}\left\{\pi_{X}(x)\right\}, \pi_{\max }^{(n)}(x):=\max _{X \in D_{n}}\left\{\pi_{X}(x)\right\}$, have been studied by Jansen et al.(1986) and more recently in greater detail in Hürlimann(1998b), Section III.5. Since there is a one-to-one correspondence between a distribution and its stoploss transform, this is (1.1) and the fact $\bar{F}_{X}(x)=-\pi_{X}{ }^{\prime}(x)$, one defines stop-loss ordered minimal and maximal random variables $X_{s, \text { min }}^{(n)}, X_{s, \text { max }}^{(n)}$ by setting for their distributions

$$
F_{s l, \min }^{(n)}(x)=1+\frac{d}{d x} \pi_{\min }^{(n)}(x), F_{s l, \max }^{(n)}(x)=1+\frac{d}{d x} \pi_{\max }^{(n)}(x) .
$$


These are extremal in the sense that $X_{s l, \min }^{(n)} \leq_{s l} X \leq_{s l} X_{s l, \max }^{(n)}$ for all $X \in D_{n}$. For $n=$ $2,3,4$, the above extremal random variables have been described completely in Hürlimann(1998b).

The Appendix, whose four Sections are numbered with roman letters, offers a self-contained derivation of the distribution functions of the maximal random variables with respect to the stochastic dominance and stop-loss order in the relevant moment spaces.

\section{THE VAR AND CVAR RISK MEASURES}

Consider a firm confronted with a risky business over some time period, and let the random variable $X$ represent the potential loss or risk the firm incurs at the end of the period. To be able to cover any loss with a high probability, the firm borrows at the beginning of the time period on the capital market the amount $E R C_{0}$, called economic risk capital. At the end of the period, the firm has to pay interest on this at the interest rate $i_{R}$. To guarantee with certainty the value of the borrowed capital at the end of the period, the firm invests $E R C_{0}$ at the risk-free interest rate $i_{f}<i_{R}$. The value of the economic risk capital at the end of the period is thus $E R C$ $=E R C_{0} \cdot\left(1+i_{f}-i_{R}\right)$. The risky business will be successful at the end of the period provided the event $\{X>E R C\}$ occurs only with a small tolerance probability.

There exist several risk management principles applied to evaluate $E R C$. Two simple methods that have been considered so far are the value-at-risk and the expected shortfall or conditional value-at-risk approach (e.g. Embrechts(1995), Arztner et al.(1997/99), Arztner(1999), Wirch(1999), Wirch and Hardy(1999), Delbaen(2000), Pflug(2000), Testuri and Uryasev(2000), Acerbi(2001), Acerbi and Tasche(2001a/b), Bertsimas et al.(2001), Hürlimann(2001b/e), Kusuoka(2001), Rockafellar and Uryasev(2000/2001), Yamai and Yoshiba(2001a/b), Yoshiba and Yamai(2001)). According to the value-at-risk method one identifies the economic risk capital with the value-at-risk of the loss setting

$$
\operatorname{VaR}_{\alpha}[X]:=Q_{X}(\alpha)
$$

where $Q_{X}(u)=\inf \left\{x \mid F_{X}(x) \geq u\right\}$ is a quantile function of $X$. This quantile represents the maximum possible loss, which is not exceeded with the (high) probability $\alpha$ (called confidence level). The expected shortfall or conditional value-at-risk method is introduced as follows. Consider the upper conditional value-at-risk $\left(\mathrm{CVaR}^{+}\right)$to the confidence level $\alpha$ :

$$
\operatorname{CVaR}_{\alpha}^{+}[X]=E\left[X \mid X>\operatorname{VaR}_{\alpha}[X]\right] .
$$

This quantity represents the conditional expected loss given the loss strictly exceeds its value-at-risk. Next, consider the $\alpha$-tail transform $X^{\alpha}$ of $X$ with distribution function

$$
F_{X^{\alpha}}(x)=\left\{\begin{array}{l}
0, x<\operatorname{VaR}_{\alpha}[X], \\
\frac{F_{X}(x)-\alpha}{1-\alpha}, x \geq \operatorname{VaR}_{\alpha}[X] .
\end{array}\right.
$$


Rockafellar and Uryasev(2001) define conditional value-at-risk (CVaR) to the confidence level $\alpha$ as expected value of the $\alpha$-tail transform, that is by

$$
\operatorname{CVaR}_{\alpha}[X]=E\left[X^{\alpha}\right] \text {. }
$$

The obtained measure is a coherent risk measure in the sense of Arztner et al. (1997/99) and coincides with $\mathrm{CVaR}^{+}$in the case of continuous distributions. Alternatively, the expected shortfall (ES) to the confidence level $\alpha$ is defined by

$$
E S_{\alpha}[X]=\frac{1}{\varepsilon} \cdot \int_{\alpha}^{1} \operatorname{VaR}[X] d u,
$$

and represents the average of the $100 \varepsilon \%$ worst losses, where $\varepsilon=1-\alpha$ denotes the loss probability. The CVaR and ES quantities coincide and satisfy a lot of equivalent formulas, as shown in Hürlimann(2001e). In the present paper the following result is used.

Proposition 2.1. One has

$$
C V a R_{\mathrm{q}}[X]=E S_{\alpha}[X]=Q_{X}(\alpha)+\frac{1}{\varepsilon} \cdot \pi_{X}\left[Q_{X}(\alpha)\right],
$$

Proof. Rearranging and making a change of variables, one obtains

$$
\begin{aligned}
& E S_{\alpha}[X]=Q_{X}(\alpha)+\frac{1}{\varepsilon} \cdot \int_{\alpha}^{1}\left(Q_{X}(u)-Q_{X}(\alpha)\right) d u=Q_{X}(\alpha)+ \\
& \frac{1}{\varepsilon} \cdot \int_{Q_{X}(\alpha)}^{\infty}\left(x-Q_{X}(\alpha)\right) d F_{X}(x)=Q_{X}(\alpha)+\frac{1}{\varepsilon} \cdot \pi_{X}\left[Q_{X}(\alpha)\right] .
\end{aligned}
$$

On the other side, by definition of CVaR, one has

$$
C V a R_{\alpha}[X]=E\left[X^{\alpha}\right]=\int_{0}^{\infty} \bar{F}_{X^{\alpha}}(x) d x-\int_{-\infty}^{0} F_{X^{\alpha}}(x) d x .
$$

Using (2.3) one obtains distinguishing between the two cases $\operatorname{VaR}_{\alpha}[X] \geq 0$ and $\operatorname{VaR}_{\alpha}[X]<0$ without difficulty that

$$
\operatorname{CVaR}_{\alpha}[X]=\operatorname{VaR}_{\alpha}[X]+\frac{1}{\varepsilon} \cdot \pi_{X}\left[\operatorname{VaR}_{\alpha}[X]\right]=\operatorname{ES}_{\alpha}[X] .
$$

Mathematically, VaR and $\mathrm{CVaR}$, which have been defined as functions of random variables, may be viewed as functionals defined on the space of probability distributions associated with these random variables. Recall that both functionals satisfy important risk-preference criteria in the economics of insurance. Indeed, the value-at-risk and conditional value-at-risk methods are consistent with ordering of risks in the sense that profit-seeking (risk averse) decision makers require higher $\mathrm{VaR}(\mathrm{CVaR})$ by increasing risk, where risk is compared using the stochastic order $\leq_{s t}$ (stop-loss order $\leq_{s l}$ ). Reciprocally, increasing VaR $(\mathrm{CVaR})$ is always coupled with higher risk (Hürlimann(2001b), Theorem 1.1). 
It is well-known that the well established VaR capital requirement may fail to be sudadditive, and thus stimulate diversification, and does not take into account the severity of an incurred adverse loss event. In contrast to this, the CVaR functional, which for continuous distributions is subadditive and scalar multiplicative, is a coherent risk measure in the sense of Arztner et al.(1997/99) and appears thus more suitable in general applications. A recent work devoted to the analytical evaluation of economic risk capital and diversification using the VaR and CVaR approaches is Hürlimann(2001d).

In practice, often only incomplete information about losses are available. For example, one knows only the range $[A, B],-\infty \leq A<B \leq \infty$, of the loss and its first few $n$ moments $\mu_{1}, \ldots, \mu_{n}$, that is $X \in D_{n}:=D_{n}\left([A, B] ; \mu_{1}, \ldots, \mu_{n}\right)$, the set of all losses taking values in the interval $[A, B]$ with fixed first $n$ moments. In this situation, Theorems 3.1,4.1 and 4.2 may be applied to determine the maximal values of VaR and CVaR over some sets $D_{n}, n=2,4$. In the notations of Section 1 one has

$\min _{X \in D_{n}}\left\{\operatorname{VaR}_{\varepsilon}[X]\right\}=\operatorname{VaR}_{\varepsilon}\left[X_{s t \min }^{(n)}\right], \max _{X \in D_{n}}\left\{\operatorname{VaR}_{\varepsilon}[X]\right\}=\operatorname{VaR}_{\varepsilon}\left[X_{s h \max }^{(n)}\right]$.

Similarly, one has

$$
\min _{X \in D_{n}}\left\{\operatorname{CVaR}_{\varepsilon}[X]\right\}=\operatorname{CVaR}_{\varepsilon}\left[X_{s l, \min }^{(n)}\right], \max _{X \in D_{n}}\left\{\operatorname{CVaR}_{\varepsilon}[X]\right\}=C V a R_{\varepsilon}\left[X_{s l, \max }^{(n)}\right]
$$

\section{The MaXimum of VAR and CVAR by KNOWN MEAN AND VARIANCE}

Applying the usual linear transform $X=\mu+\sigma Z$, the calculation of the maximum of the VaR and CVaR functionals over all $X \in D_{2}([A, B] ; \mu, \sigma)$ is reduced to the same calculation over all $Z \in D_{2}([a, b] ; \mu=0, \sigma=1)$ with $a=\frac{A-\mu}{\sigma}, b=\frac{B-\mu}{\sigma}$. Surprisingly, if the loss tolerance level is sufficiently small, these maximum values coincide.

Theorem 3.1. The maximum of $\mathrm{VaR}$ and $\mathrm{CVaR}$ for the set $D_{2}:=D_{2}([A, B] ; \mu, \sigma)$ is determined as follows:

Case 1: $\varepsilon \leq \frac{\sigma^{2}}{\sigma^{2}+(B-\mu)^{2}}: \quad \max _{X \in D_{2}}\left\{\operatorname{VaR}_{\varepsilon}[X]\right\}=\max _{X \in D_{2}}\{C \operatorname{VaR}[X]\}=B$

Case 2: $\frac{\sigma^{2}}{\sigma^{2}+(B-\mu)^{2}} \leq \varepsilon \leq \frac{(\mu-A)^{2}}{\sigma^{2}+(\mu-A)^{2}}$ :

$$
\begin{aligned}
& \max _{X \in D_{2}}\left\{\operatorname{VaR}_{\varepsilon}[X]\right\}=\max _{X \in D_{2}}\{C \operatorname{VaR}[X]\}=\mu+\sqrt{\frac{1-\varepsilon}{\varepsilon}} \cdot \sigma \\
& \max _{X \in D_{2}}\left\{\operatorname{VaR}_{\varepsilon}[X]\right\}=\mu+\frac{(\mu-A)(B-A)(1-\varepsilon)-\sigma^{2}}{(B-A) \varepsilon-(\mu-A)} \leq \\
& \max _{X \in D_{2}}\{C \operatorname{VaR}[X]\}=\mu+(\mu-A)\left(\frac{1-\varepsilon}{\varepsilon}\right)
\end{aligned}
$$$$
\text { Case 3: } \varepsilon \geq \frac{(\mu-A)^{2}}{\sigma^{2}+(\mu-A)^{2}}
$$ 
The equality in Case 3 holds if and only if $B \rightarrow \infty$.

Proof. It suffices to make calculations for the set $D_{2}([a, b] ; \mu=0, \sigma=1)$ with $a=\frac{A-\mu}{\sigma}, b=\frac{B-\mu}{\sigma}$, and to transform back the result using the relation $X=\mu+$ $\sigma Z$. The maximum of $\mathrm{VaR}$ is equal to the $(1-\varepsilon)$-quantile of $F_{s, \text { max }}^{(2)}(x)$ and is obtained without difficulty from the Table III.1. Similarly, to get the maximum of CVaR, one first calculate the $(1-\varepsilon)$-quantile of $F_{s, \max }^{(2)}(x)$ using Table IV.1, which is determined by the table:

\begin{tabular}{ll}
\hline \hline condition & $Q_{s l, \max }^{(2)}(1-\varepsilon)$ \\
\hline$\varepsilon \leq \frac{1}{1+b^{2}}$ & $\mathrm{~b}$ \\
$\frac{1}{1+b^{2}}<\varepsilon \leq \frac{a^{2}}{1+a^{2}}$ & $\frac{1}{2} \cdot \frac{1-2 \varepsilon}{\sqrt{\varepsilon(1-\varepsilon)}}$ \\
$\varepsilon>\frac{a^{2}}{1+a^{2}}$ & $\mathrm{a}$ \\
\hline \hline
\end{tabular}

Inserting this into the formula (obtained from (2.8) and (2.6))

$$
\max _{X \in D_{2}}\{C \operatorname{VaR}[X]\}=Q_{s l, \text { max }}^{(2)}(1-\varepsilon)+\frac{1}{\varepsilon} \cdot \pi_{\max }^{(2)}\left[Q_{s l, \max }^{(2)}(1-\varepsilon)\right]
$$

yields the desired result. Use that in Case 1 one has $\pi_{\max }^{(2)}(b)=0$, in Case 2 the decreasing function $\frac{1}{2} \cdot \frac{1-2 \varepsilon}{\sqrt{\varepsilon(1-\varepsilon)}} \in\left[\frac{1}{2}(a+\bar{a}), \frac{1}{2}(b+\bar{b})\right]$, and in Case $3 \pi_{\max }^{(2)}(a)=-a$. If $b<\infty$ the inequality in Case 3 is strict because $\varepsilon>\frac{a^{2}}{1+a^{2}}$.

Example 3.1. To illustrate, consider a firm whose loss and profit function is known to belong to $D_{2}=D_{2}([0, B] ; \mu, \sigma)$ for some unknown finite constant $B$, which has to be determined. Suppose the firm operates on a coherent basis and sets its required risk capital equal to CVaR. According to Theorem 3.1 the maximum value of required $\mathrm{CVaR}$ equals

$$
\max _{X \in D_{2}}\left\{C V a R_{\varepsilon}[X]\right\}=\left\{\begin{array}{l}
B, \quad \varepsilon \leq \frac{\sigma^{2}}{\sigma^{2}+(B-\mu)^{2}}, \\
\mu+\sqrt{\frac{1-\varepsilon}{\varepsilon}} \cdot \sigma, \frac{\sigma^{2}}{\sigma^{2}+(B-\mu)^{2}}<\varepsilon \leq \frac{1}{1+k^{2}} \\
\left(1+\frac{1-\varepsilon}{\varepsilon}\right) \cdot \mu, \varepsilon>\frac{1}{1+k^{2}}
\end{array}\right.
$$

where $k=\frac{\sigma}{\mu}$ is the coefficient of variation. Using the inequality (II.5), which guarantees the existence of random variables with parameters $\mu, \sigma$, one must 
have $1+\left(\frac{-\mu}{\sigma}\right)\left(\frac{B-\mu}{\sigma}\right) \leq 0$, hence $B \geq\left(1+k^{2}\right) \cdot \mu$. If the firm desires to operate at the lowest possible cost (for reasons of competitiveness), then the firm must cut its loss and profit at $B=\left(1+k^{2}\right) \cdot \mu$ (buying reinsurance or/and financial options) and the maximum required risk capital is equal to

$$
\max _{X \in D_{2}}\left\{\operatorname{CVaR}_{\varepsilon}[X]\right\}=\left\{\begin{array}{l}
\left(1+k^{2}\right) \cdot \mu \quad \varepsilon \leq \frac{1}{1+k^{2}}, \\
\left(1+\frac{1-\varepsilon}{\varepsilon}\right) \cdot \mu, \quad \varepsilon>\frac{1}{1+k^{2}} .
\end{array}\right.
$$

This very simple formula might be useful in connection with the problem of firm mergers discussed by Arztner(1999), p. 22. It is interesting and striking to note that the amount $\left(1+k^{2}\right) \cdot \mu$ identifies with the Karlsruhe price introduced by Heilmann(1987). It also identifies with the limiting CAPM fair premium in Hürlimann(1994), Theorem 4.1, and for large $k$ it is approximately equal to the Hardy-Littlewood price introduced in Hürlimann(1998a). Are these relationships mere coincidence or does there exist a more theoretical explanation for them?

\section{KNOWN MEAN, VARIANCE, SKEWNESS AND KURTOSIS}

Though it is possible to determine the Chebyshev-Markov and the stop-loss ordered extremal random variables for the sets $D_{n}\left([A, B] ; \mu_{1}, \ldots, \mu_{n}\right)$ up to $n=4$ for arbitrary intervals $[A, B],-\infty \leq A<B \leq \infty$, which is done in Hürlimann(1998b), we will focus on the set $D_{4}:=D_{4}\left((-\infty, \infty) ; \mu, \sigma, \gamma, \gamma_{2}\right)$ of all random variables defined on $(-\infty, \infty)$ with known mean $\mu$, variance $\sigma^{2}$, skewness $\gamma$ and kurtosis $\gamma_{2}$. This does not only yield the simplest explicit analytical results, but is universally applicable in the sense that any random variable can be thought of as defined on $(-\infty, \infty)$. The possible loss in precision due to this restriction is compensated by a higher mathematical tractability resulting in a wider practical usefulness. The following auxiliary parameters will be used throughout:

$$
\Delta=2+\gamma_{2}-\gamma^{2}, \quad c=\frac{1}{2}\left(\gamma-\sqrt{4+\gamma^{2}}\right), \quad \bar{c}=-c^{-1}=\frac{1}{2}\left(\gamma+\sqrt{4+\gamma^{2}}\right) .
$$

Their properties and role is described in the Appendix, Section II. The maximum of the VaR and CVaR functionals are explicitly determined by the analytical expressions in the next two results. As a corollary, we show that these expressions coincide provided the loss tolerance level is sufficiently small (generalization of Theorem 3.1).

Theorem 4.1. The maximum of VaR for the set $D_{4}$ equals

$$
\max _{X \in D_{4}}\{\operatorname{VaR}[X]\}=\mu+x_{\varepsilon} \cdot \sigma,
$$


where the $(1-\varepsilon)$-quantile $x_{\varepsilon}$ of the standardized Chebyshev-Markov maximal distribution $F_{s t, \text { max }}^{(4)}(x)$ is obtained from the following equations:

Case 1: $\quad \varepsilon \leq p(\bar{c})=\frac{1}{2}\left(1-\frac{\gamma}{\sqrt{4+\gamma^{2}}}\right), \quad p\left(x_{\varepsilon}\right)=\varepsilon$,

Case 2: $\quad \varepsilon>p(\bar{c})$,

$$
p\left(\psi\left(x_{\varepsilon}\right)\right)=1-\varepsilon
$$

where the functions $\psi(x)$ and $p(x)$ are defined in (II.12) and (II.14) of the Appendix.

Proof. The standardized Chebyshev-Markov maximal distribution functions $F_{s, \max }^{(4)}(x)$ are described in Table III.2. Since $\lim _{x \rightarrow c} p(\psi(x))=0$ one must distinguish between $x_{\varepsilon} \geq \bar{c}$ (Case 1) and $c \leq x_{\varepsilon} \leq \bar{c}$ (Case 2). For $x \geq \bar{c}$ one notes that the function $p(x)$ in (II.14) is monotone decreasing. Therefore, the condition $x_{\varepsilon} \geq \bar{c}$ for Case 1 is equivalent with the inequality $\varepsilon=p\left(x_{\varepsilon}\right) \leq p(\bar{c})$. Using (II.13) one has $q(\bar{c})=0$. Inserted in (II.14) one gets $p(\bar{c})=\frac{1}{1+(\bar{c})^{2}}=\frac{1}{2}\left(1-\frac{\gamma}{\sqrt{4+\gamma^{2}}}\right)$. This shows Case 1. Since $\psi(\bar{c})=c$ one has $p(\psi(\bar{c}))=1-p(\bar{c})$, hence $F_{s, \text { max }}^{(4)}(x)$ is continuous and strictly increasing. Case 2 follows immediately from Table III.2.

Theorem 4.2. The maximum of $C V a R$ for the set $D_{4}$ equals

$$
\max _{X \in D_{4}}\{C \operatorname{VaR}[X]\}=\mu+\left\{d\left(y_{\varepsilon}\right)+\frac{1}{\varepsilon} \cdot\left(\pi_{\max }^{(4)} \cdot d\right)\left(y_{\varepsilon}\right)\right\} \cdot \sigma,
$$

where the $(1-\varepsilon)$-quantile $d\left(y_{\varepsilon}\right)$ of the standardized stop-loss ordered maximal distribution $F_{s l, \max }^{(4)}(x)$ is obtained from the following equations:

Case 1: $\quad \varepsilon \leq p(\bar{c}), \quad p\left(y_{\varepsilon}\right)=\varepsilon$,

Case 2: $\quad \varepsilon>p(\bar{c}), \quad p\left(y_{\varepsilon}\right)=1-\varepsilon$

with $p(x)$ from (II.14), $d(x)$ from (IV.1), and $\left(\pi_{\max }^{(4)} \circ d\right)(x)$ from Table IV.2.

Proof. The standardized stop-loss ordered maximal distribution function $F_{s l, \text { max }}^{(4)}$ $(d(x))$ is described in Table IV.1. For $x \leq c$ the function $p(x)$ is monotone increasing, and for $x \geq \bar{c}$ it is monotone decreasing. Since $p(c)=1-p(\bar{c})$ the distribution $F_{s, \text { max }}^{(4)}(d(x))$ is continuous and strictly increasing. The formulas for the $(1-\varepsilon)$-quantile $d\left(y_{\varepsilon}\right)$ follow immediately. Setting $Z=X_{s l, \max }^{(4)}$, one obtains the standardized maximal CVaR from (2.8) and (2.6), that is $C V a R_{\varepsilon}[Z]=Q_{Z}(1-\varepsilon)+$ $\frac{1}{\varepsilon} \cdot \pi_{Z}\left[Q_{Z}(1-\varepsilon)\right]=d\left(y_{\varepsilon}\right)+\frac{1}{\varepsilon} \cdot \pi_{\max }^{(4)}\left(d\left(y_{\varepsilon}\right)\right)$. The result follows by transformation using the relation $X=\mu+\sigma Z$.

Corollary 4.1. If the loss tolerance level satisfies the inequality $\varepsilon \leq p(\bar{c})$, then the maximum of VaR and CVaR coincide. More precisely, with $p\left(x_{\varepsilon}\right)=\varepsilon$ one has

$$
\max _{X \in D_{4}}\left\{\operatorname{VaR}_{\varepsilon}[X]\right\}=\max _{X \in D_{4}}\{C \operatorname{VaR}[X]\}=\mu+x_{\varepsilon} \cdot \sigma,
$$


Proof. Comparing Theorem 4.1 and 4.2 one notes that in Case 1 one has $y_{\varepsilon}=x_{\varepsilon}$. But, from Table IV.2, one has in Case 1 that $\left(\pi_{\max }^{(4)} \circ d\right)(x)=p(x) \cdot(x-d(x))$. Inserting into (4.3) and using that $p\left(y_{\varepsilon}\right)=\varepsilon$, one obtains (4.4).

Example 4.1. To get (4.4) it suffices to solve the biquadratic equation $q(x)^{2}+$ $\Delta x^{2}-\left(\frac{1-\varepsilon}{\varepsilon}\right) \Delta=0$. For a symmetric distribution with $\gamma=0$ one gets the explicit formula

$$
x_{\varepsilon}=\frac{\sqrt{2}}{2} \cdot \sqrt{\sqrt{\gamma_{2}^{2}+4\left(\frac{1-\varepsilon}{\varepsilon}\right)\left(\gamma_{2}+3\right)-\frac{4}{\varepsilon}}-\gamma_{2}} .
$$

If additionally $\gamma_{2}=0$ (parameters of a normal distribution), one has $x_{\varepsilon}=\sqrt[4]{\frac{2-3 \varepsilon}{\varepsilon}}$.

Remark 4.1. In Case 2 equality between (4.2) and (4.3) does not hold. With $y_{\varepsilon}=\psi\left(x_{\varepsilon}\right)$ one obtains inserting the relation $\left(\pi_{\max }^{(4)} \circ d\right)(x)=p(x) \cdot(d(x)-x)-d(x)$ from Table IV.2 into (4.3):

$$
\max _{X \in D_{4}}\left\{C V a R_{\varepsilon}[X]\right\}=\mu-\left(\frac{1-\varepsilon}{\varepsilon}\right) \psi\left(x_{\varepsilon}\right) \cdot \sigma .
$$

For example, if $\gamma=\gamma_{2}=0, \varepsilon=0.6$, (Case 2), $\mu=1.15, \sigma=0.25$, one gets

$$
\max _{X \in D_{4}}\left\{\operatorname{VaR}_{\varepsilon}[X]\right\}=1.3425<\max _{X \in D_{4}}\left\{C V a R_{\varepsilon}[X]\right\}=1.3482 .
$$

One expects that strict inequality is always valid in Case 2.

\section{THE MAXIMUM CVAR FOR PORTFOLIOS UNDER INCOMPLETE INFORMATION}

An important but complex problem is the evaluation of VaR and CVaR for the aggregate loss of portfolios. Let $X=\left(X_{1}, \ldots, X_{n}\right)$ be a portfolio of multivariate losses, where the marginal losses $X_{i}$ have distributions $F_{i}(x), i=1, \ldots, n$. Suppose one is interested in the maximum VaR and CVaR for the aggregate loss $S(X)=X_{1}+\ldots+X_{n}$ whenever $X \in D\left(F_{1}, \ldots, F_{n}\right)$ belongs to the set of all multivariate losses with given marginals $F_{i}(x)$. The evaluation of the maximum VaR of a portfolio with fixed margins is related to Kolmogorov's problem treated among others in Makarov(1981), Rüschendorf(1982), Frank et al.(1987), Denuit et al.(1999), Durrleman et al.(2000), Luciano and Marena(2001), Cossette et al.(2001), Embrechts et al.(2001). The determination of the maximum CVaR of portfolios by fixed margins is much simpler. As shown in Hürlimann (2001b), Theorems 2.2 and 2.3 (also Dhaene et al.(2000), Corollary 6 and Theorem 8 ), for absolutely continuous distributions one has

$$
\max _{X \in D\left(F_{1}, \ldots F_{n}\right)}\left\{C V a R_{\varepsilon}[S(X)]\right\}=C V a R_{\varepsilon}\left[S\left(X^{c}\right)\right]=\sum_{i=1}^{n} C V a R_{\varepsilon}\left[X_{i}\right]
$$

where $\boldsymbol{X}^{c}=\left(F_{1}^{-1}(U), \ldots, F_{n}^{-1}(U)\right) \in D\left(F_{1}, \ldots, F_{n}\right)$, with $U$ uniformly distributed on $[0,1]$, is the portfolio with mutually comonotonic components. This result 
means that comonotonicity, which displays the strongest possible dependence structure, corresponds to the riskiest portfolio under all portfolios with the same marginal losses and requires the maximum CVaR under all these portfolios. Furthermore, the maximum $\mathrm{CVaR}$ is an additive functional. In contrast to this, the solution to Makarov's problem shows that the maximum VaR is not attained at $X^{c}=\left(F_{1}^{-1}(U), \ldots, F_{n}^{-1}(U)\right)$. In the comonotonic situation one has only the additive relation

$$
\operatorname{VaR}_{\varepsilon}\left[S\left(X^{c}\right)\right]=\sum_{i=1}^{n} \operatorname{VaR}_{\varepsilon}\left[X_{i}\right]
$$

The result (5.1) yields a simple recipe for the calculation of the maximum CVaR for the aggregate loss of portfolios given incomplete information about the marginal losses, say $X_{i} \in D_{m}^{i}:=D_{m}^{i}\left((-\infty, \infty) ; \mu_{1}^{i}, \ldots, \mu_{m}^{i}\right), m \in\{2,4\}, i=1, \ldots, n$, for which it is known that the corresponding stop-loss ordered extremal random variables $X_{i, s l, \max }^{(m)}$ have absolutely continuous distributions (Section 3 for $m=2$, Section 4, proof of Theorem 4.2 for $m=4$ ).

Theorem 5.1. The maximum CVaR for the aggregate loss of a portfolio $X=$ $\left(X_{1}, \ldots, X_{n}\right) \in D\left(F_{1}, \ldots, F_{n}\right)$ with $X_{i} \in D_{m}^{i}, m \in\{2,4\}$, is determined as follows:

$$
\max _{X \in D\left(F_{1}, \ldots F_{n}\right) X_{i} \in D_{m}^{i}}\{C \operatorname{VaR}[S(X)]\}=\sum_{i=1}^{n} \max _{X_{i} \in D_{m}^{i}}\left\{C \operatorname{VaR}\left[X_{i}\right]\right\} .
$$

\section{SOME NUMERICAL COMPARISONS}

It appears instructive to illustrate and compare results of VaR and CVaR calculations at some typical situations of general interest.

Table 6.1 compares VaR and CVaR for two-parameter distributions with fixed mean and variance by varying loss probability. Under these conditions it is impossible to compare distributions in the stochastic dominance and stoploss order. As a consequence, Theorem 3.1 does not apply and there exists no simple rule, which allows for comparison of VaR and CVaR values. Also, there is no simple dependence on tail thickness and right-tail risk. If one identifies higher right-tail risk with a higher-degree stop-loss order, as done in Hürlimann $(2000)$, then it is possible to rank distributions with fixed mean and variance. Up to the normal and the Benktander II distribution and some few exceptions, which depend on the coefficient of variation, the distributions of Table 6.1 are ranked by increasing higher-degree stop-loss order (Hürlimann(2000), Theorem 4.1 and Proposition 5.3). One sees immediately that VaR and CVaR are not consistent with an increase in right-tail risk. For $\varepsilon \geq 0.05$ the distributions with highest right-tail risk require less $\mathrm{VaR}$ and CVaR than a normally distributed risk. Up to the normal, there is a big difference between VaR and CVaR, and the results for $\mathrm{VaR}$ are quite counterintuitive. For example, if $\varepsilon \geq 0.05$ the VaR of the Pareto is smaller than the VaR of the normal, though it is well-known that the Pareto is able to model very dangerous insurance risks. For $\varepsilon \leq 0.01$ the $\mathrm{CVaR}$ of a normal distribution behaves more according to our 
intuition than VaR, but is not consistent with tail-thickness and right-tail risk, even for small loss probabilities of the magnitude $\varepsilon=0.001$.

TABLE 6.1

VAR AND CVAR COMPARISONS FOR TWO-PARAMETER DISTRIBUTIONS WITH FIXED MEAN $\mu=1$ AND VARIANCE $\sigma^{2}=3$ BY VARYING $\varepsilon$

\begin{tabular}{lccccccccccc}
\hline \multirow{2}{*}{ distribution } & \multicolumn{2}{c}{$\boldsymbol{\varepsilon}=\mathbf{0 . 1 0}$} & \multicolumn{2}{c}{$\boldsymbol{\varepsilon}=\mathbf{0 . 0 5}$} & \multicolumn{2}{c}{$\boldsymbol{\varepsilon}=\mathbf{0 . 0 1}$} & \multicolumn{2}{c}{$=\mathbf{0 . 0 0 2}$} & \multicolumn{2}{c}{$\boldsymbol{\varepsilon}=\mathbf{0 . 0 0 1}$} \\
\cline { 2 - 12 } & $\mathbf{V a R}$ & $\mathbf{C V a R}$ & $\mathbf{V a R}$ & $\mathbf{C V a R}$ & $\mathbf{V a R}$ & $\mathbf{C V a R}$ & $\mathbf{V a R}$ & $\mathbf{C V a R}$ & VaR & $\mathbf{C V a R}$ \\
\hline normal & 3.22 & 4.04 & 3.85 & 4.57 & 5.03 & 5.62 & 5.99 & 6.49 & 6.35 & 6.83 \\
gamma & 2.91 & 5.20 & 4.42 & 6.84 & 8.30 & 10.89 & 12.46 & 15.15 & 14.30 & 17.02 \\
inverse Gaussian & 2.42 & 4.98 & 3.91 & 6.91 & 8.62 & 12.39 & 14.61 & 18.88 & 17.47 & 21.90 \\
Weibull & 2.67 & 5.05 & 4.11 & 6.82 & 8.35 & 11.73 & 13.67 & 17.66 & 16.27 & 20.50 \\
exp. inv. Gaussian & 2.52 & 4.94 & 3.92 & 6.77 & 8.31 & 12.16 & 14.31 & 19.15 & 17.38 & 22.65 \\
log-normal & 2.26 & 4.59 & 3.47 & 6.40 & 7.74 & 12.53 & 14.81 & 22.25 & 19.02 & 27.89 \\
Benktander II & 2.35 & 4.68 & 3.58 & 6.48 & 7.85 & 12.44 & 14.71 & 21.56 & 18.69 & 26.71 \\
Benktander I & 2.33 & 4.59 & 3.51 & 6.35 & 7.62 & 12.28 & 14.44 & 21.88 & 18.55 & 27.57 \\
Pareto & 2.31 & 4.46 & 3.43 & 6.14 & 7.28 & 11.92 & 13.87 & 21.81 & 18.00 & 28.00 \\
para-logistic & 2.05 & 3.87 & 2.92 & 5.31 & 6.13 & 10.73 & 12.29 & 21.25 & 16.49 & 28.45 \\
inverse gamma & 1.88 & 3.62 & 2.68 & 5.02 & 5.78 & 10.43 & 11.94 & 21.20 & 16.21 & 28.67 \\
log-logistic & 1.87 & 3.47 & 2.61 & 4.75 & 5.42 & 9.77 & 11.11 & 19.97 & 15.11 & 27.17 \\
inv. para-logistic & 1.78 & 3.31 & 2.48 & 4.55 & 5.18 & 9.44 & 10.72 & 19.50 & 14.65 & 26.64 \\
inverse Weibull & 1.71 & 3.04 & 2.37 & 4.15 & 4.98 & 8.50 & 10.37 & 17.40 & 14.22 & 23.68 \\
log-Laplace & 1.65 & 3.06 & 2.27 & 4.20 & 4.76 & 8.81 & 9.97 & 18.45 & 13.71 & 25.37 \\
maximum & 6.20 & 8.55 & 18.23 & 39.69 & 55.74 \\
\hline \hline
\end{tabular}

Table 6.2 compares $\mathrm{VaR}, \mathrm{CVaR}$ and the maximum $\mathrm{CVaR}$ for a log-normal distribution by varying mean $\mu$ and standard deviation $\sigma$ but fixed $\varepsilon=0.05$. The values of the skewness and kurtosis coefficients are those of the log-normal and given by

$$
\gamma=k \cdot\left(3+k^{2}\right), \gamma_{2}=k^{2} \cdot\left(16+15 k^{2}+6 k^{4}+k^{6}\right), k=\frac{\sigma}{\mu} .
$$

The displayed distributions are able to model one-year accumulated returns of equity indices in financial markets. Compared to the obtained exact value, the maximum $\mathrm{CVaR}$ contains a relatively small implicit margin.

TABLE 6.2

VAR AND CVAR COMPARISONS FOR A LOG-NORMAL DISTRIBUTION WITH FIXED $\varepsilon=0.05$

\begin{tabular}{lccccc}
\hline \hline $\boldsymbol{\mu}$ & $\boldsymbol{\sigma}$ & VaR & CVaR & $\max _{\boldsymbol{X} \in D_{\mathbf{4}}}\left\{\boldsymbol{C V a R _ { \varepsilon } [ X ] \}}\right.$ & implicit margin (in \%) \\
\hline 1.10 & 0.15 & 1.363 & 1.444 & 1.501 & 3.9 \\
& 0.20 & 1.456 & 1.574 & 1.651 & 4.9 \\
& 0.25 & 1.552 & 1.710 & 1.810 & 5.9 \\
& 0.30 & 1.649 & 1.854 & 1.981 & 6.9 \\
\hline
\end{tabular}




\begin{tabular}{lccccc}
\hline $\boldsymbol{\mu}$ & $\boldsymbol{\sigma}$ & VaR & CVaR & $\max _{\boldsymbol{X} \in \boldsymbol{D}_{\mathbf{4}}}\left\{\boldsymbol{C V a R _ { \varepsilon } [ \boldsymbol { X } ] \}}\right.$ & implicit margin (in \%) \\
\hline 1.15 & 0.15 & 1.412 & 1.492 & 1.548 & 3.7 \\
& 0.20 & 1.505 & 1.621 & 1.698 & 4.7 \\
& 0.25 & 1.600 & 1.756 & 1.855 & 5.6 \\
& 0.30 & 1.697 & 1.898 & 2.024 & 6.6 \\
1.20 & 0.15 & 1.461 & 1.541 & 1.598 & 3.7 \\
& 0.20 & 1.554 & 1.669 & 1.745 & 4.6 \\
& 0.25 & 1.649 & 1.803 & 1.901 & 5.5 \\
1.25 & 0.30 & 1.745 & 1.943 & 2.067 & 6.4 \\
& 0.15 & 1.511 & 1.590 & 1.646 & 3.6 \\
& 0.20 & 1.603 & 1.716 & 1.792 & 5.4 \\
& 0.25 & 1.698 & 1.849 & 1.947 & 6.1 \\
\hline \hline
\end{tabular}

The comparisons for one-year accumulated returns of equity indices is pursued in Table 6.3. By fixed $\varepsilon=0.05$, time-dependent comparisons are made between VaR, CVaR and the maximum CVaR assuming respectively a normal and a log-normal distribution with time dependent mean and standard deviation described by

$$
\mu_{t}=(1.08)^{t}, \sigma_{t}=\sqrt{e^{\beta^{2} t}-1} \cdot \mu_{t}, \beta=\sqrt{\ln \left\{1+\left(\frac{0.2}{1.08}\right)^{2}\right\}}=0.184 \text {. }
$$

This corresponds to a Black-Scholes model, whose log-normal distribution has time-dependent parameters $\left[\ln (1.08)-\frac{1}{2} \beta^{2}\right] \cdot t$ and $\beta \cdot \sqrt{t}$, where 1.08 is the one-year accumulated risk-free rate and $\beta=0.184$ is the one-year volatility. In the long-term, the VaR and especially the $\mathrm{CVaR}$ are underestimated using a normal assumption. The maximum CVaR is calculated using the skewness and kurtosis of a log-normal. The implicit margin contained in this distribution-free upper bound increases over the time, which agrees with our intuition of increasing uncertainty by increasing time horizon.

TABLE 6.3

TIME-DEPENDENT VAR AND CVAR COMPARISONS FOR A NORMAL AND LOG-NORMAL DISTRIBUTION WITH FIXED $\varepsilon=0.05$

\begin{tabular}{|c|c|c|c|c|c|c|}
\hline \multirow[b]{2}{*}{$\mathbf{t}$} & \multicolumn{2}{|c|}{ normal } & \multicolumn{2}{|c|}{ log-normal } & \multirow[b]{2}{*}{$\max _{X \in D_{\mathbf{4}}}\left\{\boldsymbol{C V a R _ { \varepsilon }}[X]\right\}$} & \multirow[b]{2}{*}{$\begin{array}{l}\text { implicit margin } \\
\quad \text { (in \%) }\end{array}$} \\
\hline & VaR & CVaR & VaR & CVaR & & \\
\hline 1 & 1.409 & 1.493 & 1.436 & 1.555 & 1.632 & 5.0 \\
\hline 2 & 1.673 & 1.802 & 1.729 & 1.936 & 2.066 & 6.7 \\
\hline 3 & 1.936 & 2.107 & 2.021 & 2.325 & 2.516 & 8.2 \\
\hline 4 & 2.211 & 2.427 & 2.327 & 2.740 & 3.007 & 9.7 \\
\hline 5 & 2.505 & 2.768 & 2.654 & 3.190 & 3.550 & 11.3 \\
\hline
\end{tabular}




\begin{tabular}{rcccccc}
\hline \hline \multicolumn{7}{c}{ normal } \\
\cline { 2 - 6 } & VaR & CVaR & VaR & CVaR & $\max _{\boldsymbol{X} \in \boldsymbol{D}_{\mathbf{4}}}\left\{\boldsymbol{C V a R _ { \varepsilon } [ \boldsymbol { X } ] \}}\right.$ & $\begin{array}{c}\text { implicit margin } \\
\text { (in \%) }\end{array}$ \\
\hline 6 & 2.823 & 3.137 & 3.006 & 3.683 & 4.155 & 12.8 \\
7 & 3.168 & 3.538 & 3.387 & 4.224 & 4.832 & 14.4 \\
8 & 3.545 & 3.975 & 3.800 & 4.820 & 5.590 & 16.0 \\
9 & 3.957 & 4.454 & 4.250 & 5.477 & 6.438 & 17.6 \\
10 & 4.408 & 4.979 & 4.741 & 6.201 & 7.385 & 19.1 \\
\hline \hline
\end{tabular}

Next, Table 6.4 displays the dependence of VaR, CVaR and the maximum CVaR upon the kurtosis parameter for two important symmetric distributions, namely the normal inverted gamma mixture and the symmetric double Weibull distribution recently considered in Hürlimann(2001c). The first distribution is conditional on $\theta$ normally distributed with mean $\mu$ and variance $\theta^{-1}$, where $\theta$ follows a conjugate gamma prior $\Gamma\left(\frac{1}{2} c^{2}, \alpha\right), \alpha>1$. The corresponding density, distribution and stop-loss transform are described by the formulas

$$
\begin{aligned}
& f(x)=\frac{1}{B\left(\alpha, \frac{1}{2}\right)} \cdot \frac{1}{c}\left[\frac{c^{2}}{c^{2}+(x-\mu)^{2}}\right]^{\alpha+\frac{1}{2}}, \quad B(a, b)=\frac{\Gamma(a) \Gamma(b)}{\Gamma(a+b)}, \\
& F(x)= \begin{cases}\frac{1}{2}\left[1+\beta\left(\frac{1}{2}, \alpha ; \frac{(x-\mu)^{2}}{c^{2}+(x-\mu)^{2}}\right)\right], & x \geq \mu, \\
\frac{1}{2}\left[1-\beta\left(\frac{1}{2}, \alpha ; \frac{(x-\mu)^{2}}{c^{2}+(x-\mu)^{2}}\right)\right], & x \leq \mu,\end{cases} \\
& \pi(x)=\frac{c^{2}+(x-\mu)^{2}}{2 \alpha-1} \cdot f(x)-(x-\mu) \cdot(1-F(x)),
\end{aligned}
$$

where $c=\sigma \cdot \sqrt{2(\alpha-1)}, \sigma$ the standard deviation, and $\beta(a, b ; x)$ is a beta density with parameters $a$ and $b$. The kurtosis exists only for $\alpha>2$ and is given by

$$
\gamma_{2}=\frac{3}{\alpha-2}, \quad \alpha>2
$$

The standardized distribution of $t_{\alpha}=\sqrt{2(\alpha-1)} \cdot\left(\frac{X-\mu}{c}\right)$ is a generalized Student $\mathrm{t}$ and converges for $\alpha \rightarrow \infty$ to a standard normal distribution with kurtosis $\gamma_{2}=0$. The density, distribution and stop-loss transform of a standardized symmetric double Weibull distribution with parameter $\alpha>0$ are given by the formulas

$$
\begin{aligned}
& f(z)=\frac{1}{2} \alpha \lambda_{\alpha}|z|^{\alpha-1} \exp \left(-\lambda_{\alpha}|z|^{\alpha}\right), \quad \lambda_{\alpha}=\Gamma\left(1+\frac{2}{\alpha}\right)^{\frac{\alpha}{2}}, \\
& F(z)= \begin{cases}\frac{1}{2} \exp \left(-\lambda_{\alpha}|z|^{\alpha}\right), \quad z \leq 0, \\
1-\frac{1}{2} \exp \left(-\lambda_{\alpha}|z|^{\alpha}\right), \quad z \geq 0,\end{cases} \\
& \pi(z)=\frac{1}{2} \lambda_{\alpha}^{-\frac{1}{\alpha}} \Gamma\left(1+\frac{1}{\alpha}\right) \cdot\left\{1-\Gamma\left(1+\frac{1}{\alpha} ; \lambda_{\alpha}|z|^{\alpha}\right)\right\}-z \cdot(1-F(z)),
\end{aligned}
$$


where $\Gamma(\beta ; x)$ is a gamma distribution with shape parameter $\beta$. The kurtosis of this distribution, which equals

$$
\gamma_{2}=\frac{\Gamma\left(1+\frac{4}{\alpha}\right)}{\Gamma\left(1+\frac{2}{\alpha}\right)^{2}}-3,
$$

covers the whole range $[-2, \infty)$ of possible values. The dependence of VaR upon the kurtosis is counterintuitive. Leptokurtic distributions require a decreasing VaR by increasing kurtosis. On the other hand, the dependence of CVaR behaves in accordance with our intuition of higher risk by increasing kurtosis. The implicit margin in the maximum CVaR is moderate but increases by increasing kurtosis. It is smaller for the symmetric double Weibull model.

TABLE 6.4

VAR AND CVAR DEPENDENCE ON KURTOSIS BY FIXED $\varepsilon=0.05, \mu=1.08, \sigma=0.2$

\begin{tabular}{|c|c|c|c|c|c|}
\hline distribution & kurtosis & VaR & CVaR & $\max _{X \in D_{4}}\{C \operatorname{VaR}[X]\}$ & $\begin{array}{l}\text { implicit margin } \\
\text { (in \%) }\end{array}$ \\
\hline \multirow[t]{5}{*}{ symmetric NIG } & 12 & 1.388 & 1.531 & 1.754 & 14.6 \\
\hline & 6 & 1.392 & 1.528 & 1.702 & 11.4 \\
\hline & 3 & 1.397 & 1.523 & 1.657 & 8.8 \\
\hline & 1 & 1.404 & 1.511 & 1.609 & 6.5 \\
\hline & 0.5 & 1.407 & 1.504 & 1.593 & 5.9 \\
\hline normal & 0 & 1.409 & 1.493 & 1.573 & 5.4 \\
\hline \multirow[t]{6}{*}{ symmetric DW } & 0 & 1.402 & 1.489 & 1.573 & 5.6 \\
\hline & 0.5 & 1.406 & 1.505 & 1.593 & 5.9 \\
\hline & 1 & 1.407 & 1.517 & 1.609 & 6.1 \\
\hline & 3 & 1.403 & 1.547 & 1.657 & 7.1 \\
\hline & 6 & 1.397 & 1.568 & 1.702 & 8.5 \\
\hline & 12 & 1.378 & 1.582 & 1.754 & 10.8 \\
\hline
\end{tabular}

Finally, in Table 6.5 we compare VaR, CVaR and maximum CVaR for a normal and gamma approximation to a classical compound Poisson distribution, which models the aggregate loss of an insurance portfolio. If the expected value of the individual claims is one unit, and if $\lambda$ represents the expected number of claims of the insurance portfolio, then the relevant parameters of the gamma approximation are for some constant $c$ equal to

$$
\mu=\lambda, \quad k=\frac{c}{\sqrt{\lambda}}, \quad \gamma=2 k, \quad \gamma_{2}=6 k^{2}
$$

The constant $c=1.85$ in our illustration corresponds to the risk of a typical life insurance portfolio. For $\lambda \leq 1000$ the difference between VaR and CVaR is not negligible. The normal approximation underestimates systematically VaR and CVaR of the gamma model, especially for small values of $\lambda$. The maximum CVaR for the set $D_{4}$ for large $\lambda$ is quite close to the CVaR of the gamma, while for small $\lambda$ it is close to the maximum CVaR for the set $D_{2}$. 
TABLE 6.5

VAR AND CVAR COMPARISONS FOR A GAMMA AND A NORMAL BY FIXED $\varepsilon=0.05$

\begin{tabular}{lccccccc}
\hline \hline & \multicolumn{2}{c}{ VaR } & \multicolumn{2}{c}{$C V a R$} & $\max _{X \in D_{4}}\left\{C V a R_{\varepsilon}[X]\right\}$ & $\begin{array}{c}\text { implicit } \\
\text { margin }\end{array}$ & $\max _{\boldsymbol{X} \in D_{2}}\left\{C V a R_{\varepsilon}[X]\right\}$ \\
\cline { 2 - 7 } & normal & gamma & normal & gamma & & \\
\hline 1 & 4.04 & 4.61 & 4.82 & 7.31 & 9.00 & 23.1 & 9.06 \\
2 & 6.30 & 7.27 & 7.40 & 10.33 & 12.34 & 19.4 & 13.40 \\
5 & 11.80 & 13.14 & 13.53 & 16.87 & 19.11 & 13.3 & 23.03 \\
10 & 19.62 & 21.15 & 22.07 & 25.58 & 28.17 & 10.1 & 35.50 \\
20 & 33.61 & 35.26 & 37.07 & 40.70 & 43.95 & 8.0 & 56.06 \\
50 & 71.52 & 73.28 & 76.98 & 80.72 & 85.59 & 6.0 & 107.0 \\
100 & 130.4 & 132.2 & 138.2 & 141.9 & 148.8 & 4.9 & 180.6 \\
200 & 243.0 & 244.9 & 254.0 & 257.8 & 267.6 & 3.8 & 314.0 \\
500 & 568.0 & 569.9 & 585.3 & 589.2 & 605.0 & 2.7 & 680.3 \\
1000 & 1096 & 1098 & 1121 & 1125 & 1147 & 2.0 & 1255 \\
10000 & 10304 & 10306 & 10382 & 10385 & 10459 & 0.7 & 10806 \\
100000 & 100962 & 100964 & 101207 & 101211 & 101446 & 0.2 & 102550 \\
\hline \hline
\end{tabular}

Appendix: Stochastic and stop-loss ordered bounds by known moments to order four

To construct the stochastic and stop-loss ordered maximal distribution functions $F_{s, \text { max }}^{(n)}(x)$ and $F_{s l, \max }^{(n)}(x)$ for the moments spaces $D_{n}:=D_{n}\left([A, B] ; \mu_{1}, \ldots, \mu_{n}\right)$, it is necessary to solve the optimization problems $\max _{X \in D_{n}}\left\{E\left[I_{[x, \infty)}(X)\right]\right\}$ and $\max _{X \in D_{n}}\left\{E\left[(X-x)_{+}\right]\right\}$, where $I_{[x, \infty)}(z)$ is the Heaviside indicator function, defined to be 0 if $z<x$ and 1 otherwise. Both belong to the class of extremal problems $\max _{X \in D_{n}}\{E[f(X)]\}$, where $f(x)$ is a piecewise linear function, and which have been extensively studied in Hürlimann(1997a/b/98b). A general approach to solve these problems is the well-known polynomial majorant method, which consists to bound $f(x)$ by some polynomial $q(x)$ of degree less or equal to $n$, and to construct a finite atomic random variable $Z \in D_{n}$ such that all atoms of $f(Z)$ are simultaneously atoms of $q(Z)$. Indeed, suppose $q(x)$ and $Z$ have been found such that $\operatorname{Pr}(q(Z)$ $=f(Z))=1$ and $q(x) \geq f(x)$ for all $x \in[A, B]$. Then the expected value $E[q(Z)]$ $=E[f(Z)]$ depends only on the first moments $\mu_{1}, \ldots, \mu_{n}$, and thus necessarily $Z$ maximizes $E[f(X)]$ over all $X \in D_{n}$. A brief outline of the Appendix follows.

In Section I we derive in a first step finite exhaustive lists of all polynomials of a given degree, which can be used to construct polynomial majorants for $f(x)=I_{[t, \infty)}(x)$ and $f(x)=(x-d)_{+}$. A second step in the construction of best bounds for expected values by given range and known moments of higher order consists in a detailed analysis of the algebraic moment problem for finite atomic random variables. The most useful results are based on the explicit analytical structure of bi- and triatomic random variables by given range and known moments up to order four as presented in Section II. Then, in Section III we 
derive the stochastic ordered bounds and in Section IV the corresponding stoploss ordered bounds.

\section{Polynomial majorants for the Heaviside indicator and the stop-loss functions}

The Heaviside indicator function $f(x)=I_{[t, \infty)}(x)$ and the stop-loss function $f(x)$ $=(x-d)_{+}, d$ the deductible, belong to the class of piecewise linear functions $f(x)$ on an interval $I=[a, b],-\infty \leq a<b \leq \infty$. For these simple but most important prototypes, one can decompose $I$ into two disjoint adjacent pieces such that $I=I_{1} \cup I_{2}$, and the function of interest is a linear function $f(x)=\ell_{i}(x)=\alpha_{i}+\beta_{i} x$ on each piece $I_{i}, i=1,2$. If $q(x)$ is a polynomial of degree $n \geq 2$, then $q(x)-f(x)$ is a piecewise polynomial function of degree $n$, which is denoted by $Q(x)$ and which coincides on $I_{i}$ with the polynomial $Q_{i}(x)=q(x)-\ell_{i}(x)$ of degree $n$. For the construction of polynomial majorants $q(x) \geq f(x)$ on $I$, one can restrict the attention to finite atomic random variables $X$ with support $\left\{x_{0}=a, x_{1}, \ldots\right.$, $\left.x_{r}, x_{r+1}=b\right\} \subset I$ such that $\operatorname{Pr}(q(X)=f(X))$ (e.g. Karlin and Studden(1966), Theorem XII.2.1). By convention, if $a=-\infty$ then $x_{0}=a$ is removed from the support and if $b=\infty$ then $x_{r+1}=b$ is removed. In general, the fact that $x_{0}=a$ or/and $x_{r+1}=b$ does no belong to the support of $X$ is technically achieved by setting the corresponding probabilities equal to zero. If an atom of $X$, say $x_{k}$, is an interior point of some $I_{i}$, then it must be a double zero of $Q_{i}(x)$. Indeed $q(x) \geq \ell_{i}(x)$ for $x \in I_{i}$ is only fulfilled if the line $\ell_{i}(x)$ is tangent to $q(x)$ at $x_{k}$, that is $q^{\prime}\left(x_{k}\right)=\ell_{i}^{\prime}\left(x_{k}\right)$. From this simple observation, one derives finite exhaustive lists of all polynomials of a given degree, which are used to construct polynomial majorants (Tables I.1 and I.2).

Consider first the indicator function $f(x)=I_{[t, \infty)}(x)$. We decompose the interval $I=[a, b]$ into the pieces $I_{1}=[a, t], I_{2}=[t, b]$, such that $f(x)=\ell_{1}(x)=0$ on $I_{1}$ and $f(x)=\ell_{2}(x)=1$ on $I_{2}$. For a fixed $m \in\{1, \ldots, r\}$ the atom $x_{m}=t$ belongs always to the support of a maximizing finite atomic random variable $X$. We show that a polynomial majorant of fixed degree is always among the finite many possibilities listed in Table I.1.

Proposition I.1. Let $\left\{x_{0}=a, x_{1}, \ldots, x_{m}=t, \ldots, x_{r}, x_{r+1}=b\right\}, x_{r}<x_{s}$ for $r<s$, $m \in\{1, \ldots, r\}$, be the support of a random variable $X$ on $I$, and let $q(x)$ be a polynomial majorant such that $\operatorname{Pr}(q(X)=f(X))=1$ and $q(x) \geq f(x)$ on $I$. Then $q(x)$ is uniquely determined by the conditions in Table I.1.

TABLE I. 1

POLYNOMIAL MAJORANTS FOR THE HEAVISIDE INDICATOR FUNCTION

\begin{tabular}{lllll}
\hline \hline case & support with $\boldsymbol{x}_{\boldsymbol{m}}=\boldsymbol{t}$ & $\boldsymbol{Q}_{j}\left(\boldsymbol{x}_{\boldsymbol{i}}\right)=\mathbf{0 , j = \mathbf { 1 } , \mathbf { 2 }}$ & $\boldsymbol{Q}_{j}^{\prime}\left(\boldsymbol{x}_{\boldsymbol{i}}\right)=\mathbf{0 , j} \boldsymbol{j} \mathbf{1 , 2}$ & $\operatorname{deg} \boldsymbol{q}(\boldsymbol{x})$ \\
\hline$(1)$ & $\left\{a, x_{1}, \ldots, x_{r}, b\right\}$ & $i=0, \ldots, r+1$ & $i \neq 0, m, r+1$ & $2 r$ \\
$(2)$ & $\left\{a, x_{1}, \ldots, x_{r}\right\}$ & $i=0, \ldots, r$ & $i \neq 0, m$ & $2 r-1$ \\
$(3)$ & $\left\{x_{1}, \ldots, x_{r}, b\right\}$ & $i=1, \ldots, r+1$ & $i \neq m, r+1$ & $2 r-1$ \\
$(4)$ & $\left\{x_{1}, \ldots, x_{r}\right\}$ & $i=1, \ldots, r$ & $i \neq m$ & $2 r-2$ \\
\hline \hline
\end{tabular}


Proof. Restrict the attention to case(1) (the other cases are shown similarly). One must show the existence of a unique polynomial $q(x)$ of degree $n=2 r$ as in Figure I.1.

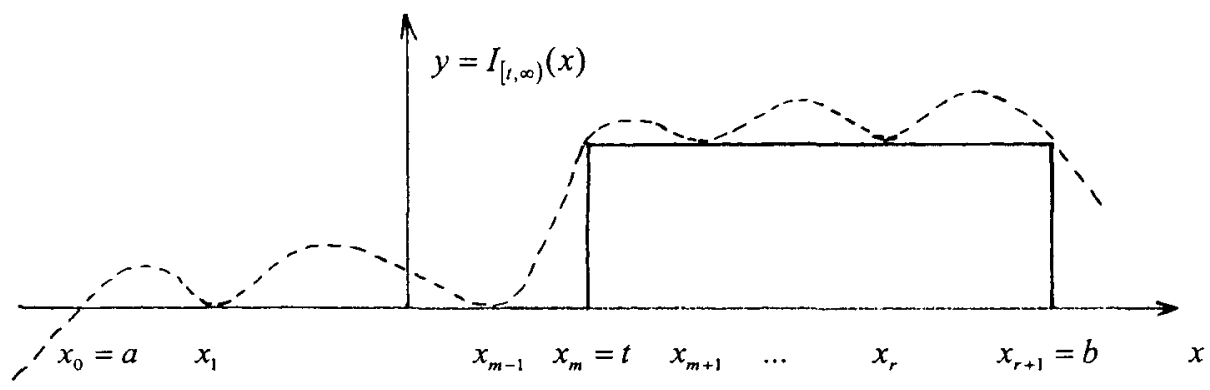

Figure I.1: Polynomial majorant $q(x) \geq I_{[\{, \infty)}(x), \quad x \in[a, b]$

Consider the unique polynomial $q(x)$ of degree $n=2 r$ such that

$$
q\left(x_{i}\right)=\left\{\begin{array}{ll}
0, & i=0, \ldots, m-1 \\
1, & i=m, \ldots, r+1
\end{array}, \quad q^{\prime}\left(x_{i}\right)=0, \quad i \neq 0, m, r+1 .\right.
$$

By definition of $Q_{j}(x), j=1,2$, the conditions of Table I.1 under case (1) are fulfilled. By the theorem of Rolle, the derivative $q^{\prime}(x)$ vanishes at least once on each of the $r$ subintervals $\left(x_{i}, x_{i+1}\right), 0 \leq i \leq r, i \neq m-1$. In fact one has exactly $(r-1)+r=n-1$ zeros of $q^{\prime}(x)$ on $I$. Furthermore, one has $q^{\prime}(x)>0$ on $\left(x_{m-1}, x_{m}\right)$. More precisely, one has $q^{\prime}(x)>0$ on $\left(x_{m-1}, x_{m}\right)$ because $q\left(x_{m-1}\right)$ $=0<q\left(x_{m}\right)=1$. It follows that $q(x)$ is local minimal at all $x_{i}, i \neq 0, m, r+1$, and local maximal between each consecutive minima, as well as in the intervals $\left(a, x_{1}\right)$ and $\left(x_{r}, b\right)$. These properties imply the inequality $q(x) \geq I_{[t, \infty)}(x)$, $x \in[a, b]$.

Consider now the stop-loss function $f(x)=(x-d)_{+}, d \in[a, b]$ the deductible, $I_{1}=[a, d], I_{2}=[d, b]$. Then the stop-loss function $f(x)$ may be viewed as the piecewise linear function defined by $f(x)=\ell_{1}(x)=0$ on $I_{1}, f(x)=\ell_{2}(x)=x-d$ on $I_{2}$. By convention $m \in\{1, \ldots, r\}$ is fixed such that $x_{m}<d<x_{m+1}$. A polynomial majorant of fixed degree for $f(x)$ belongs always to one of the finitely many types listed in Table I. 2 below. The notations $q(x ; \xi, d)$ and $Q_{j}(x ; \xi, d)$ mean that these functions depend upon the parameter vector $\xi=\left(x_{0}, \ldots, x_{r+1}\right)$ and the deductible $d$.

Proposition I.2. Let $\left\{x_{0}=a, x_{1}, \ldots, x_{r}, x_{r+1}=b\right\}, x_{r}<x_{s}$ for $r<s, x_{m}<d<x_{m+1}$, be the ordered support of a random variable $X$ defined on $I$, and let $q(x)$ be a polynomial majorant such that $\operatorname{Pr}(q(X)=f(X))=1$ and $q(x) \geq f(x)$ on $I$. Then $q(x)$ is a polynomial uniquely determined by the conditions in Table I.2. 
TABLE I.2

POLYNOMIAL MAJORANTS FOR THE STOP-LOSS FUNCTION

\begin{tabular}{llllll}
\hline \hline case & $\begin{array}{l}\text { support } \\
\boldsymbol{x}_{\boldsymbol{m}}<\boldsymbol{d}<\boldsymbol{x}_{\boldsymbol{m}+1}\end{array}$ & $\begin{array}{l}Q_{j}\left(\boldsymbol{x}_{\boldsymbol{i}}\right) \mathbf{0}, \\
\boldsymbol{j}=\mathbf{1 , 2}\end{array}$ & $\begin{array}{l}\boldsymbol{Q}_{j}^{\prime}\left(\boldsymbol{x}_{\boldsymbol{i}}\right)=\mathbf{0}, \\
\boldsymbol{j}=\mathbf{1 , 2}\end{array}$ & $\operatorname{deg} \boldsymbol{q}(\boldsymbol{x})$ & $\begin{array}{l}\text { condition on } \\
\text { deductible } \boldsymbol{d}\end{array}$ \\
\hline (la) & $\left\{a, x_{1}, \ldots, x_{r}, b\right\}$ & $i=0, \ldots, r+1$ & $i=1, \ldots, r-1$ & $2 r$ & $Q_{2}^{\prime}\left(x_{r} ; \xi, d\right)=0$ \\
(1b) & $\left\{a, x_{1}, \ldots, x_{r}, b\right\}$ & $i=0, \ldots, r+1$ & $i=1, \ldots, r$ & $2 r+1$ & $Q_{1}(y ; \xi, d)=0, y \in(-\infty, a]$ \\
(1c) & $\left\{a, x_{1}, \ldots, x_{r}, b\right\}$ & $i=0, \ldots, r+1$ & $i=1, \ldots, r$ & $2 r+1$ & $Q_{2}(z ; \xi, d)=0, z \in[b, \infty)$ \\
(2a) & $\left\{x_{1}, \ldots, x_{r}, b\right\}$ & $i=1, \ldots, r+1$ & $i=1, \ldots, r$ & $2 r$ & $Q_{2}(z ; \xi, d)=0, z \in[b, \infty)$ \\
(2b) & $\left\{x_{1}, \ldots, x_{r}, b\right\}$ & $i=1, \ldots, r+1$ & $i=2, \ldots, r$ & $2 r-1$ & $Q_{1}^{\prime}\left(x_{1} ; \xi, d\right)=0$, \\
(3a) & $\left\{a, x_{1}, \ldots, x_{r}\right\}$ & $i=0, \ldots, r$ & $i=1, \ldots, r$ & $2 r$ & $Q_{1}(y ; \xi, d)=0, y \in(-\infty, a]$ \\
(3b) & $\left\{a, x_{1}, \ldots, x_{r}\right\}$ & $i=0, \ldots, r$ & $i=1, \ldots, r-1$ & $2 r-1$ & $Q_{2}^{\prime}\left(x_{r} ; \xi, d\right)=0$ \\
(4a) & $\left\{x_{1}, \ldots, x_{r}\right\}$ & $i=1, \ldots, r$ & $i=1, \ldots, r-1$ & $2 r-2$ & $Q_{2}^{\prime}\left(x_{r} ; \xi, d\right)=0$ \\
(4b) & $\left\{x_{1}, \ldots, x_{r}\right\}$ & $i=1, \ldots, r$ & $i=1, \ldots, r$ & $2 r-1$ & $Q_{1}(y ; \xi, d)=0, y \in(-\infty, a]$ \\
(4c) & $\left\{x_{1}, \ldots, x_{r}\right\}$ & $i=1, \ldots, r$ & $i=1, \ldots, r$ & $2 r-1$ & $Q_{2}(z ; \xi, d)=0, z \in[b, \infty)$ \\
\hline \hline
\end{tabular}

Proof. There are essentially two typical cases for which a proof is required, say (1a) and (1b). The other cases are shown by the same method and omitted for this reason. In case (1a) one shows the existence of a unique polynomial $q(x)$ of degree $n=2 r$ as in Figure I.2. Consider the unique polynomial $q(x)$ of degree $n=2 r$ such that

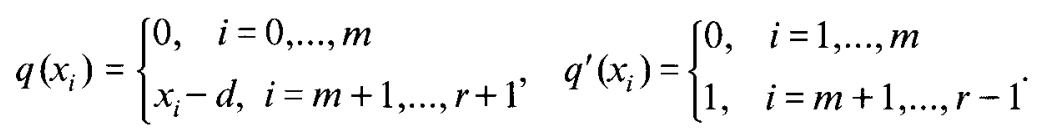

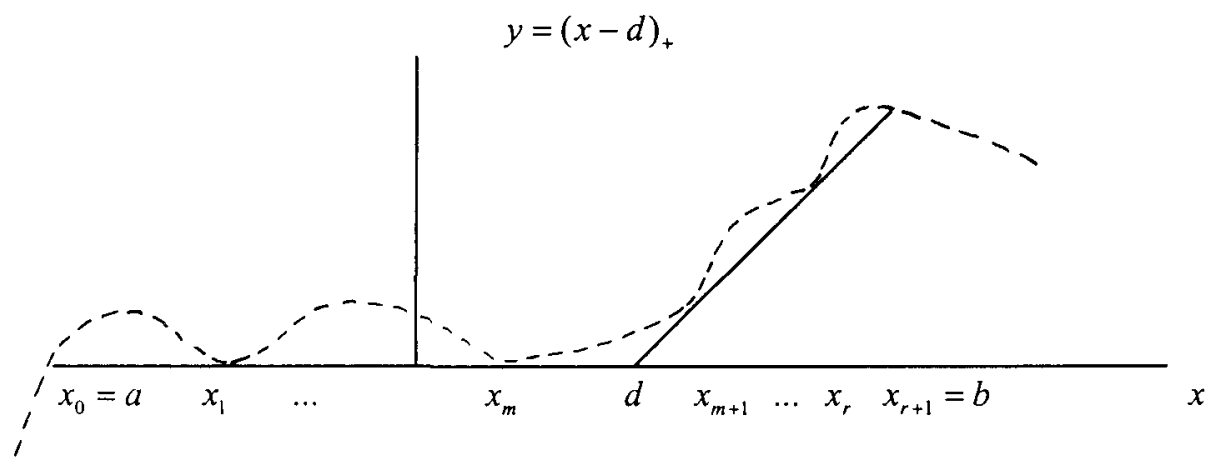

Figure I.2: Polynomial majorant $q(x) \geq(x-d)_{+}, x \in[a, b]$, case (la).

By definition of $Q_{j}(x), j=1,2$, the conditions of Table 1.2 under case (1a) are fulfilled. In order that $q(x) \geq(x-d)_{+}$, the line $\ell_{2}(x)=x-d$ must be tangent of $q(x)$ at the remaining atom $x=x_{r}$, that is $q^{\prime}\left(x_{r}\right)=1$ or $Q_{2}^{\prime}\left(x_{r} ; \xi, d\right)=0$. This condition is an implicit equation for the deductible $d$ and restricts its range of variation. The theorem of Rolle implies the following facts: 
(i) $Q_{1}^{\prime}(x)=q^{\prime}(x)$ vanishes at least once on each of the $m$ subintervals $\left(x_{i}, x_{i+1}\right)$, $i=1, \ldots, m$.

(ii) $Q_{2}^{\prime}(x)=q^{\prime}(x)-1$ vanishes at least once on each of the $r-m$ subintervals, $\left(x_{i}, x_{i+1}\right), i=m+1, \ldots, r$.

(iii) $Q_{1}^{\prime}(x) \neq 0$ on $\left(x_{m}, d\right]$ and $Q_{2}^{\prime}(x) \neq 0$ on $\left[d, x_{m+1}\right)$. More precisely one has $Q_{1}^{\prime}(x)>0$ on $\left(x_{m}, d\right]$ since $Q_{1}\left(x_{m}\right)=0<Q_{1}\left(x_{m+1}\right)=x_{m+1}-d$, and $Q_{2}^{\prime}(x)<0$ on $\left[d, x_{m+1}\right)$ since $Q_{2}\left(x_{m}\right)=d-x_{m}>0=Q_{2}\left(x_{m+1}\right)$.

In particular there are exactly $n-1$ zeros of $q^{\prime}(x)$ on $I$. It follows that $Q_{1}(x)$ is local minimal at all $x_{i}, i=1, \ldots, m$, and local maximal between each consecutive minima, as well as in the interval $\left(a, x_{1}\right)$. Similarly $Q_{2}(x)$ is local minimal at all $x_{i}, i=m+1, \ldots, r$, and local maximal between each consecutive minima, as well as in the interval $\left(x_{r}, b\right)$. These properties imply that $Q_{1}(x) \geq 0$ on $I_{1}$ and $Q_{2}(x) \geq 0$ on $I_{2}$, which together means that $q(x) \geq(x-d)_{+}$on $I_{1} \cup I_{2}=I$. In case (1b) one shows the existence of a unique polynomial $q(x)$ of degree $n=2 r+1$ as in Figure I.3, where $y$ is a further zero of $q(x)$ in $(-\infty, a]$.

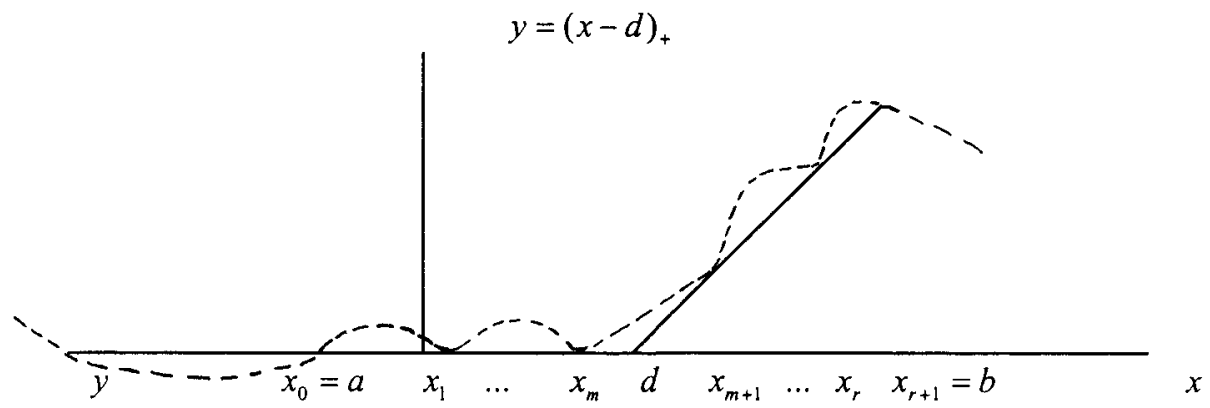

Figure 1.3: Polynomial majorant $q(x) \geq(x-d)_{+}, x \in[a, b]$, case (1b)

Consider the unique polynomial $q(x)$ of degree $n=2 r+1$ such that

$$
q\left(x_{i}\right)=\left\{\begin{array}{l}
0, \quad i=0, \ldots, m \\
x_{i}-d, i=m+1, \ldots, r+1
\end{array}, \quad q^{\prime}\left(x_{i}\right)=\left\{\begin{array}{ll}
0, & i=1, \ldots, m \\
1, & i=m+1, \ldots, r
\end{array} .\right.\right.
$$

By definition of $Q_{j}(x), j=1,2$, the conditions of Table I.2 under case (1b) are fulfilled. In order that $q(x)$ is a polynomial of odd degree, there must exist a further zero $y$ of $q(x)$ in $(-\infty, a]$, which yields the implicit equation $Q_{1}(y ; \xi, d)=0$, for the deductible $d$. The rest of the proof follows similarly to case (la).

\section{Structure of finite atomic random variables by known moments to order four}

The algebraic-analytical structure of the required sets of finite atomic random variables by given range and known moments to order four is implicit in Jansen 
et al.(1986), Section 2 . However, by considering without loss of generality only standardized random variables, much calculation can been simplified and some results find improvement. Our derivation uses the solution of the algebraic moment problem by Mammana(1954), which itself is a direct application of the mathematical theory of orthogonal polynomials.

Consider a real random variable $X$ with an infinite number of non-zero finite moments $\mu_{k}=E\left[X^{k}\right], k=0,1,2, \ldots$ By convention one sets $p_{0}(x)=\mu_{0}=1$.

Definition II.1. The orthogonal polynomial of degree $n \geq 1$ with respect to the moment structure $\left\{\mu_{k}\right\}_{k=0, \ldots, 2 n-1}$, also called orthogonal polynomial with respect to $X$, is the unique monic polynomial $p_{n}(x)$ of degree $n$, which satisfies the $n$ linear expected value equations

$$
E\left[p_{n}(X) \cdot X^{i}\right]=0, \quad i=0,1, \ldots, n-1 .
$$

Note that the terminology "orthogonal" refers to the scalar product induced by the expectation operator $\langle X, Y\rangle=E[X Y]$, where $X, Y$ are random variables for which this quantity exists. The orthogonal polynomials are also called classical Chebyshev polynomials.

Given the first $2 n-1$ moments of some real random variable $X$, the algebraic moment problem of order $n(\mathrm{AMP}(n))$ asks for the existence and construction of a finite atomic random variable with ordered support $\left\{x_{1}, \ldots, x_{n}\right\}$ such that $x_{1}<x_{2}<\ldots<x_{n}$, and probabilities $\left\{p_{1}, \ldots, p_{n}\right\}$ such that the system of non-linear equations

$$
\sum_{i=1}^{n} p_{i} x_{i}^{k}=\mu_{k}, \quad k=0, \ldots, 2 n-1,
$$

is solvable. For computational purposes it suffices to know that if a solution exists, then the atoms of the random variable solving $\operatorname{AMP}(n)$ must be identical with the distinct real zeros of the orthogonal polynomial of degree $n$, as shown by the following precise recipe.

Lemma II.1. (Mammana(1954)) Given are positive numbers $p_{1}, \ldots, p_{n}$ and real distinct numbers $x_{1}<x_{2}<\ldots<x_{n}$ such that the system AMP $(n)$ is solvable. Then the $x_{i}{ }^{\prime} s$ are the distinct real zeros of the orthogonal polynomial of degree $n$, that is $p_{n}\left(x_{i}\right)=0, i=1, \ldots, n$, and

$$
p_{i}=\prod_{j \neq i}\left(x_{i}-x_{j}\right)^{-1} \cdot E\left[\prod_{j \neq i}\left(Z-x_{j}\right)\right], \quad i=1, \ldots, n,
$$

where $Z$ denotes the discrete random variable with support $\left\{x_{1}, \ldots, x_{n}\right\}$ and probabilities $\left\{p_{1}, \ldots, p_{n}\right\}$ defined by $\operatorname{AMP}(n)$.

As a next preliminary step, it is important to state the conditions under which there exist random variables on a finite interval with given moments to order four (e.g. Jansen et al.(1986)). A recent general proof for the existence of moment spaces, or equivalently for the existence of random variables with 
known moments up to a given order, is in De Vylder(1996), part II, Chapter 3.3. From now on the attention is restricted to the set $D(a, b)$ of all standard random variables with support $[a, b],-\infty \leq a<b \leq \infty$, mean $\mu=0$ and standard deviation $\sigma=1$.

Lemma II.2. (Moment inequalities) There exist non-degenerate standard random variables in $D(a, b)$ if and only if the following two conditions hold:

$$
\begin{aligned}
& a<0<b \quad \text { (inequalities on the mean } \mu=0 \text { ) } \\
& 1+a b \leq 0 \text { (inequality on the variance } \sigma^{2}=1 \text { ) }
\end{aligned}
$$

There exist non-degenerate standard random variables in $D(-\infty, \infty)$ with given moments to order four if and only if the following inequality holds:

$$
\Delta=\gamma_{2}-\gamma^{2}+2 \geq 0
$$

where for $X \in D(-\infty, \infty)$ the parameter $\gamma=E\left[X^{3}\right]$ denotes the skewness and $\gamma_{2}=$ $E\left[X^{4}\right]-3$ denotes the kurtosis.

Proof. The first two inequalities follow by taking expectations in the following random inequalities, which are valid with probability one for all $X \in D(a, b)$ :

$$
\begin{aligned}
& a \leq X \leq b, \\
& (b-X)(X-a) \geq 0,
\end{aligned}
$$

where for a non-degenerate random variable, the inequalities in (II.4') must be strict. The inequality (II.6) follows from the inequality

$$
(X-c)^{2} \cdot(X-\bar{c})^{2} \geq 0
$$

where $c=\frac{1}{2}\left(\gamma-\sqrt{4+\gamma^{2}}\right), \quad \bar{c}=-c^{-1}=\frac{1}{2}\left(\gamma+\sqrt{4+\gamma^{2}}\right)$.

Remark II.1. The inequality (II.6) between skewness and kurtosis has been known for a long time (e.g. Pearson(1916), Wilkins(1944) and Guiard(1980)).

Theorem II.1. (Characterization of standard biatomic random variables on $[a, b]$ ) Suppose that $a<0<b$ and $1+a b \leq 0$. The support $\left\{x_{1}, x_{2}\right\}, x_{1}<x_{2}$ and probabilities $\left\{p_{1}, p_{2}\right\}$ of a biatomic standard random variables $X \in D(a, b)$ are uniquely determined by

$$
\begin{aligned}
& x_{1}=x \in[a, \bar{b}], \quad x_{2}=\bar{x} \in[\bar{a}, b], \\
& p_{1}=\frac{1}{1+x^{2}}, \quad p_{2}=\frac{x^{2}}{1+x^{2}},
\end{aligned}
$$

where the upper bar denotes a strictly increasing involution function such that $\overline{\bar{x}}=x$, which maps $x \neq 0$ to $\bar{x}=-1 / x$. 
Proof. By Lemma II.2 the conditions (II.4) and (II.5) are required. By Lemma II.1 (solution of $\operatorname{AMP}(2)$ ) the atoms $x_{1}, x_{2}$ are the distinct real zeros of the standard quadratic orthogonal polynomial $p_{2}(x)=x^{2}-\gamma x-1$, where $\gamma$ is a variable skewness parameter. The Vietà formulas imply the relations $x_{1}+x_{2}=\gamma, x_{1} x_{2}=-1$. Setting $x_{1}=x$ one must have $x_{2}=\bar{x}$ and $x<0<\bar{x}$. If $x \in(\bar{b}, 0)$ then $\bar{x} \in(b, \infty)$ and the support $\{x, \bar{x}\}$ is not feasible. Therefore one must have $x \in(a, \bar{b})$, which determines uniquely the support $\{x, \bar{x}\}$. The formulas (II.8) for the probabilities follows from (II.3), that is from

$$
p_{1}=E\left[\frac{X-\bar{x}}{\bar{x}-\bar{x}}\right], \quad p_{2}=E\left[\frac{X-x}{\bar{x}-x}\right] .
$$

Proposition II.2. (Characterization of standard triatomic random variables on $(-\infty, \infty)$ with skewness $\gamma$ and kurtosis $\left.\gamma_{2}\right)$ Suppose that $\Delta=\gamma_{2}-\gamma^{2}+2 \geq 0$. The support $\left\{x_{1}, x_{2}, x_{3}\right\}, x_{1}<x_{2}<x_{3}$, and probabilities $\left\{p_{1}, p_{2}, p_{3}\right\}$ of a triatomic random variable $X \in D(-\infty, \infty)$ with skewness $\gamma$ and kurtosis $\gamma_{2}$, are uniquely determined as follows:

$$
\begin{gathered}
x_{1}=x \in(-\infty, c], \quad x_{2}=\varphi(x, \psi(x)) \in[c, \bar{c}], \quad x_{3}=\psi(x) \in[\bar{c}, \infty), \\
p_{i}=p\left(x_{i}\right), \quad i=1,2,3,
\end{gathered}
$$

where $c=\frac{1}{2}\left(\gamma-\sqrt{4+\gamma^{2}}\right), \quad \bar{c}=-c^{-1}=\frac{1}{2}\left(\gamma+\sqrt{4+\gamma^{2}}\right)$, and the functions $\varphi(u, v)$, $\psi(u)$, and $p(u)$ are defined by

$$
\begin{gathered}
\varphi(u, v)=\frac{\gamma-u-v}{1+u v}, \\
\psi(u)=\frac{1}{2}\left(\frac{A(u)-\sqrt{A(u)^{2}+4 q(u) B(u)}}{q(u)}\right), \\
A(u)=\gamma q(u)+\Delta u, \quad B(u)=\Delta+q(u), q(u)=1+\gamma u-u^{2}, \\
p(u)=\frac{\Delta}{q(u)^{2}+\Delta\left(1+u^{2}\right)} .
\end{gathered}
$$

Furthermore, the function $\psi(u)$ defines a strictly increasing involution such that $\psi^{2}(u)=u$, which maps the interval $(-\infty, c]$ to the interval $[\bar{c}, \infty)$.

Proof. By Lemma II.2 the condition (II.6) is required. By Lemma II.1 (solution of AMP(3)) the atoms are the distinct real zeros of the standard cubic orthogonal polynomial of degree three $p_{3}(x)$, which satisfies the three linear expected value equations

$$
E\left[X^{i} p_{3}(X)\right]=0, \quad i=0,1,2 .
$$

The condition

$$
E\left[p_{3}(X)\right]=E\left[\left(X-x_{1}\right)\left(X-x_{2}\right)\left(X-x_{3}\right)\right]=\gamma-\left(x_{1}+x_{2}+x_{3}\right)-x_{1} x_{2} x_{3}=0
$$


implies the relationship $x_{2}=\varphi\left(x_{1}, x_{3}\right)$. Inserted into the condition $E\left[X p_{3}(X)\right]=E\left[X\left(X-x_{1}\right)\left(X-x_{2}\right)\left(X-x_{3}\right)\right]=\gamma_{2}+3-\left(x_{1}+x_{2}+x_{3}\right) \gamma+$ $\left(x_{1} x_{2}+x_{1} x_{3}+x_{2} x_{3}\right)=0$,

one obtains that $x_{3}$ is solution of the quadratic equation $q\left(x_{1}\right) x_{3}^{2}-A\left(x_{1}\right) x_{3}-$ $B\left(x_{1}\right)=0$, hence $x_{3}=\psi\left(x_{1}\right)$ as defined in (II.9). The probabilities take the values

$$
p_{1}=\frac{1+x_{2} x_{3}}{\left(x_{2}-x_{1}\right)\left(x_{3}-x_{1}\right)}, \quad p_{2}=\frac{-\left(1+x_{1} x_{3}\right)}{\left(x_{2}-x_{1}\right)\left(x_{3}-x_{2}\right)}, \quad p_{3}=\frac{1+x_{1} x_{2}}{\left(x_{3}-x_{1}\right)\left(x_{3}-x_{2}\right)} \text {. }
$$

Since $x_{1}<x_{2}<x_{3}$ one must have $1+x_{2} x_{3} \geq 0,1+x_{1} x_{3} \leq 0,1+x_{1} x_{2} \geq 0$. Since $x_{1} x_{3} \leq-1$ one must have $x_{1}<0<x_{3}$, hence also $\bar{x}_{3}<0<\bar{x}_{1}$. It follows that

$$
\begin{aligned}
& \bar{x}_{3} \cdot\left(1+x_{2} x_{3}\right)=\bar{x}_{3}-x_{2} \leq 0, \\
& \bar{x}_{3} \cdot\left(1+x_{1} x_{3}\right)=\bar{x}_{3}-x_{1} \geq 0, \\
& \bar{x}_{1} \cdot\left(1+x_{1} x_{2}\right)=\bar{x}_{1}-x_{2} \geq 0,
\end{aligned}
$$

which implies the inequalities $x_{1} \leq \bar{x}_{3}<0, \bar{x}_{3} \leq x_{2} \leq \bar{x}_{1}$. Since $x_{2}=\varphi\left(x_{1}, x_{3}\right)$ the second inequalities in (II.19) are equivalent with $x_{3}^{2}-\gamma x_{3}-1 \geq 0, x_{1}^{2}-\gamma x_{1}-1 \geq 0$. Since $x_{1}<0<x_{3}$ one must have $\left(x_{1}, x_{3}\right) \in(-\infty, c] x[\bar{c}, \infty)$ and $x_{2} \in[c, \bar{c}]$. This shows (II.9). To obtain (II.10), note that $x_{2}=\varphi\left(x_{1}, \psi\left(x_{1}\right)\right)$ and $x_{3}=\psi\left(x_{1}\right)$ are solutions of the quadratic equation $q\left(x_{1}\right) x^{2}-A\left(x_{1}\right) x-B\left(x_{1}\right)=0$. One calculates

$$
\begin{aligned}
& \left(x_{2}-x_{1}\right)\left(x_{3}-x_{1}\right)=x_{1}^{2}-\left(x_{2}+x_{3}\right) x_{1}+x_{2} x_{3}=x_{1}^{2}-\frac{A\left(x_{1}\right)}{q\left(x_{1}\right)} x_{1}-\frac{B\left(x_{1}\right)}{q\left(x_{1}\right)}= \\
& -\frac{q\left(x_{1}\right)^{2}+\Delta\left(1+x_{1}^{2}\right)}{q\left(x_{1}\right)}, 1+x_{2} x_{3}=-\frac{\Delta}{q\left(x_{1}\right)} .
\end{aligned}
$$

Inserted into (II.16) one gets $p_{1}=p\left(x_{1}\right)$. Making cyclic permutations of $x_{1}, x_{2}$, $x_{3}$ one obtains $p_{i}=p\left(x_{i}\right), i=2,3$.

\section{Stochastic ordered bounds}

Based on the preceding two Sections, we give an explicit proof of how to construct the stochastic ordered maximal random variables in the situations applied in the present paper.

Theorem III.1. The distribution function of the standardized (ChebyschevMarkov) stochastic ordered maximal random variable $X_{s t, \max }^{(2)}$ for the set $D_{2}:=$ $D_{2}([a, b] ; \mu=0, \sigma=1)$ is described in Table III.1.

Proof. By Table I.1, quadratic polynomial majorants $q_{x}(z)$ for the Heaviside indicator function $I_{[x, \infty)}$ are obtained either for ordered biatomic supports $\{x, \bar{x}\},\{\bar{x}, x\}$ (case (4)) or for a triatomic support $\{a, x, b\}$ (case (1)). With 
Proposition II.1, one has necessarily $x \in[a, \bar{b}]$ if $\{x, \bar{x}\}$ is the extremal support, and $x \in[\bar{a}, b]$ if $\{\bar{x}, x\}$ is the extremal support. Similarly, the triatomic support $\{a, x, b\}$ is only feasible if $x \in[\bar{b}, \bar{a}]$. These supports define standard bi- and triatomic random variables $Z_{x}$ such that $\operatorname{Pr}\left(q_{x}\left(Z_{x}\right)=I_{[x, \infty)}\left(Z_{x}\right)\right)=1$. The displayed extremal values follow from the calculation of $F_{s t, \max }^{(2)}(x)=1-E\left[I_{[x, \infty)}\left(Z_{x}\right)\right]$ for each of the three cases.

TABLE III. 1

STOCHASTIC ORDERED MAXIMAL STANDARD DISTRIBUTION FOR THE SET $D_{2}$

\begin{tabular}{lll}
\hline \hline condition & $\boldsymbol{F}_{\text {st,max }}^{(2)}(\boldsymbol{x})$ & extremal support of $Z_{x}$ \\
\hline$a \leq x \leq \bar{b}$ & 0 & $\{x, \bar{x}\}$ \\
$\bar{b} \leq x \leq \bar{a}$ & $\frac{1+b x}{(b-a)(x-a)}$ & $\{a, x, b\}$ \\
$\bar{a} \leq x<b$ & $\frac{x^{2}}{1+x^{2}}$ & $\{\bar{x}, x\}$ \\
$x=b$ & 1 & \\
\hline \hline
\end{tabular}

Theorem III.2. The distribution function of the standardized (ChebyschevMarkov) stochastic ordered maximal random variable $X_{s t, \text { max }}^{(4)}$ for the set $D_{4}:=$ $D_{4}\left((-\infty, \infty) ; \mu=0, \sigma=1, \gamma, \gamma_{2}\right)$ is described in Table III.2 with the notations of Proposition II.2.

TABLE III.2

STOCHASTIC ORDERED MAXIMAL STANDARD DISTRIBUTION FOR THE SET $D_{4}$

\begin{tabular}{lll}
\hline \hline condition & $\boldsymbol{F}_{s t, \max }^{(\mathbf{)}}(\boldsymbol{x})$ & extremal support of $\boldsymbol{Z}_{\boldsymbol{x}}$ \\
\hline$x \leq c$ & 0 & $\{x, \varphi(x, \psi(x)), \psi(x)\}$ \\
$c \leq x \leq \bar{c}$ & $p(\psi(x))$ & $\{\psi(x), x, \varphi(x, \psi(x))\}$ \\
$x \geq \bar{c}$ & $1-p(x)$ & $\{\varphi(x, \psi(x)), \psi(x), x\}$ \\
\hline \hline
\end{tabular}

Proof. By Table I.1, case (4), and Proposition II.2, one observes that biquadratic polynomial majorants $q_{x}(z) \geq I_{[x, \infty)}(z)$ can only be obtained at the ordered extremal supports displayed in Table III.2. These supports define standard triatomic random variables $Z_{x}$ such that $\operatorname{Pr}\left(q_{x}\left(Z_{x}\right)=I_{[x, \infty)}\left(Z_{x}\right)\right)=1$. The displayed extremal values follow from the calculation of $F_{s i, \max }^{(4)}(x)=1-E\left[I_{[x, \infty)}\left(Z_{x}\right)\right]$ for each of the three cases.

\section{Stop-loss ordered bounds}

The present Section contains an explicit proof of how to construct the stop-loss ordered maximal random variables in the situations applied in the present paper. 
Theorem IV.1. The maximal stop-loss transform $\pi_{\max }^{(2)}(d)=\max _{X \in D_{2}}\left\{E\left[(X-d)_{+}\right]\right\}$for the set $D_{2}:=D_{2}([a, b] ; \mu=0, \sigma=1)$ and the distribution function $F_{s l \text {, } \max }^{(2)}(x)$ of the corresponding standardized stop-loss ordered maximal random variable $X_{s l, m a x}^{(2)}$ are described in Table IV.1.

TABLE IV. 1

MAXIMAL STOP-LOSS TRANSFORM AND STOP-LOSS ORDERED MAXIMAL DISTRIBUTION FOR $D_{2}$

\begin{tabular}{llll}
\hline condition & $\boldsymbol{F}_{s, \text { max }}^{(2)}(\boldsymbol{d})$ & $\boldsymbol{\pi}_{\max }^{(2)}(\boldsymbol{d})$ & extremal support \\
\hline$a \leq d \leq \frac{1}{2}(a+\bar{a})$ & $\frac{1}{1+a^{2}}$ & $(-a) \frac{1+a d}{1+a^{2}}$ & $\{a, \bar{a}\}$ \\
$\frac{1}{2}(a+\bar{a}) \leq d \leq \frac{1}{2}(b+\bar{b})$ & $\frac{1}{2}\left(1+\frac{d}{\sqrt{1+d^{2}}}\right)$ & $\frac{1}{2}\left(\sqrt{1+d^{2}-d}\right)$ & $\left\{d-\sqrt{1+d^{2}}, d+\sqrt{1+d^{2}}\right\}$ \\
$\frac{1}{2}(b+\bar{b}) \leq d<b$ & $\frac{b^{2}}{1+b^{2}}$ & $\frac{b-d}{1+b^{2}}$ & $\{\bar{b}, b\}$ \\
$d=b$ & 1 & 0 & \\
\hline \hline
\end{tabular}

Proof. The distribution function $F_{X}(x)$ and the stop-loss transform $\pi_{X}(x)$ of a random variable $X$ are related by the relationship $F_{X}(x)=1+\pi_{X}^{\prime}(x)$. Therefore, it suffices to determine the maximal stop-loss transform. By Table I.2 quadratic polynomial majorants $q(X) \geq(X-d)_{+}, d$ the deductible of the stoploss function, can only be obtained at diatomic supports of the forms $\{a, \bar{a}\}$ (case (3a), $\{x, \bar{x}\}$ (case $(4 a))$ or $\{\bar{b}, b\}$ (case (2a)).

Case (1): extremal support $\{a, \bar{a}\}$

The unique quadratic polynomial $q(X)=q(X ; a, \bar{a}, d)$ such that $q(a)=0, q(\bar{a})=$ $\bar{a}-d, q^{\prime}(\bar{a})=1$, is given by

$$
q(X)=\frac{(d-a)(X-\bar{a})^{2}}{(\bar{a}-a)^{2}}+(X-d)
$$

Solving the condition $Q_{1}(x ; a, \bar{a}, d)=q(x ; a, \bar{a}, d)=0, x \leq a$, one finds for the deductible

$$
d=\frac{\bar{a}^{2}-a x}{2 \bar{a}-a-x}, x \leq a,
$$

which implies the desired results (note that for $x \rightarrow-\infty$ one has $d \rightarrow a$ and for $x=a$ one has $\left.d=\frac{1}{2}(a+\bar{a})\right)$.

Case (2): extremal support $\{x, \bar{x}\}$

By Proposition II.1 the ordered diatomic support $\{x, \bar{x}\}$ is feasible exactly when $x \in[a, \bar{b}]$. The unique quadratic polynomial $q(X)=q(X ; x, \bar{x}, d)$ such that $q(x)=q^{\prime}(x)=0$, is given by 


$$
q(X)=\frac{(\bar{x}-d)(X-x)^{2}}{(\bar{x}-x)^{2}} .
$$

Solving the condition $Q_{2}^{\prime}(\bar{x} ; x, \bar{x}, d)=q^{\prime}(\bar{x})-1=0$, one finds

$$
d=\frac{1}{2}(x+\bar{x}), a \leq x \leq \bar{b},
$$

from which all statements follow.

Case (3): extremal support $\{\bar{b}, b\}$

The unique quadratic polynomial $q(X)=q(X ; \bar{b}, b, d)$ such that $q(\bar{b})=q^{\prime}(\bar{b})=0$, $q(b)=b-d$, is given by

$$
q(X)=\frac{(b-d)(X-\bar{b})^{2}}{(b-\bar{b})^{2}}
$$

Solving the condition $Q_{2}(x ; \bar{b}, b, d)=q(x)-(x-d)=0, x \geq b$ one finds

$$
d=\frac{b x-\bar{b}^{2}}{x+b-2 \bar{b}}, \quad x \geq b,
$$

and the stated results are immediately checked.

Theorem IV.2. The maximal stop-loss transform $\pi_{\max }^{(4)}(d)=\max _{X \in D_{4}}\left\{E\left[(X-d)_{+}\right]\right\}$for the set $D_{4}:=D_{4}\left((-\infty, \infty) ; \mu=0, \sigma=1, \gamma, \gamma_{2}\right)$ and the distribution function $F_{s, \max }^{(4)}(d)$ of the corresponding standardized stop-loss ordered maximal random variable $X_{s l, \max }^{(4)}$ are implicitly described in Table IV.2 using the "deductible" function

$$
d(x)=\frac{1}{2} \cdot \frac{\{\varphi(x, \psi(x))-x\} \cdot\{x+\psi(x)\}+2 x \cdot\{\psi(x)-x\}}{\{\varphi(x, \psi(x))-x\}+\{\psi(x)-x\}},
$$

and the notations introduced in Proposition II.2.

TABLE IV.2

MAXIMAL STOP-LOSS TRANSFORM AND STOP-LOSS ORDERED MAXIMAL DISTRIBUTION FOR $D_{4}$

\begin{tabular}{llll}
\hline \hline condition & $\boldsymbol{F}_{s, \text { max }}^{(4)}(d(x))$ & $\boldsymbol{\pi}_{\max }^{(4)}(\boldsymbol{d}(\boldsymbol{x}))$ & extremal support of $\boldsymbol{Z}_{d(x)}$ \\
\hline$x \leq c$ & $p(x)$ & $p(x) \cdot(d(x)-x)-d(x)$ & $\{x, \varphi(x, \psi(x)), \psi(x)\}$ \\
$x \geq \bar{c}$ & $1-p(x)$ & $p(x) \cdot(x-d(x))$ & $\{\varphi(x, \psi(x)), \psi(x), x\}$ \\
\hline
\end{tabular}

Proof. Applying the chain rule of differential calculus to the identity $F_{X}(x)=$ $1+\pi_{X}{ }^{\prime}(x)$ between the distribution function and stop-loss transform of a random variable $X$, one obtains the relationship 


$$
F_{X}(d(x))=1+\frac{(\pi \circ d)^{\prime}(x)}{d^{\prime}(x)}
$$

Therefore, it suffices to determine the maximal stop-loss transform. From Table I.2, case (4a), and Proposition II.2, it follows that biquadratic polynomial majorants $q(X) \geq(X-d)_{+}$can only be constructed for supports containing the three atoms $x, \varphi(x, \psi(x)), \psi(x)$. Set $f(x)=(x-d)_{+}$and let us partition the interval $I=(-\infty, \infty)$ in the two pieces $I_{1}=(-\infty, d], I_{2}=(d, \infty]$ such that $f(x)$ $=\ell_{1}(x)=0$ on $I_{1}, f(x)=\ell_{2}(x)=x-d$ on $I_{2}$. The piecewise biquadratic function $Q(x)=q(x)-f(x)$ coincides on $I_{i}$ with the polynomial $Q_{i}(x)=q(x)-\ell_{i}(x)$, $i=1,2$. Furthermore, let $u<v<w$ represent zeros of $Q_{i}(x), i=1,2$. Two cases can occur.

Case (1): $u \in I_{1}$ is a double zero of $Q_{1}(x), v, w \in I_{2}$ are double zeros of $Q_{2}(x)$ as in the following figure:

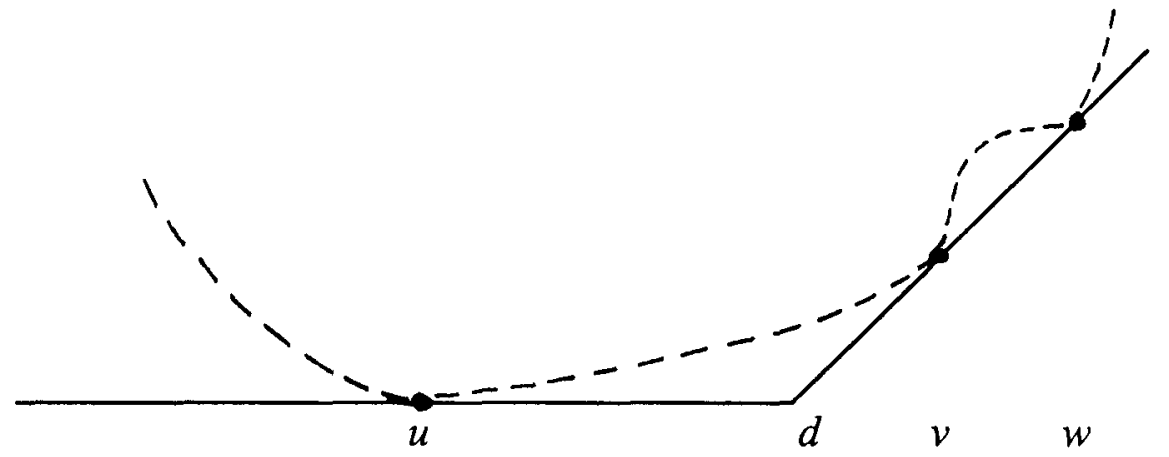

Suppose first that $u$ is a simple zero. Since a biquadratic polynomial is uniquely determined by five conditions, there exists a unique $q(x)$ with the required conditions, namely

$$
q(x)=\frac{\ell_{1}(u)-\ell_{2}(u)}{(v-u)^{2}(w-u)^{2}}(x-v)^{2}(x-w)^{2}+\ell_{2}(x) .
$$

Solving the additional $Q_{1}^{\prime}(u)=0$ condition for a double zero, one obtains

$$
d=\frac{1}{2}\left\{\frac{v w+u(v+w)-3 u^{2}}{v+w-2 u}\right\}=\frac{1}{2} \frac{(v-u)(u+w)+2(w-u) u}{(v-u)+(w-u)}
$$

The extremal support $\{u, v, w\}=\{x, \varphi(x, \psi(x)), \psi(x)\}$, feasible by (II.9) provided $x \leq c$, yields the formula for $d(x)$ as well as the maximal stop-loss transform $\pi_{\max }^{(4)}(d)=p(v) \cdot(v-d)+p(w) \cdot(w-d)=-p(u) u-(1-p(u)) d=p(u) \cdot(d-u)-d$. 
Case (2): $u, v \in I_{1}$ are double zeros of $Q_{1}(x), w \in I_{2}$, is a double zero of $Q_{2}(x)$ as in the following figure:

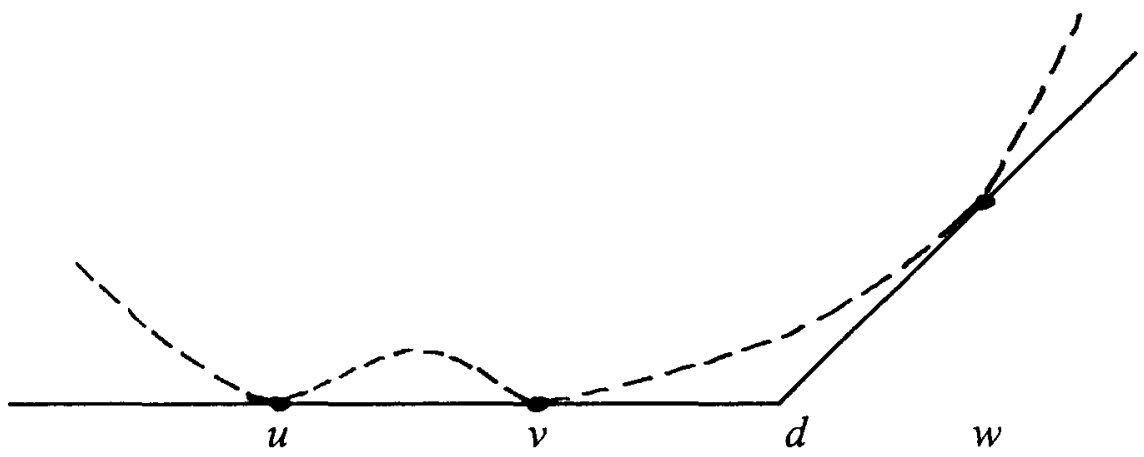

Suppose first that $w$ is a simple zero. By symmetry to case (1), the unique biquadratic polynomial with the required conditions is

$$
q(x)=\frac{\ell_{2}(w)-\ell_{1}(w)}{(w-u)^{2}(w-v)^{2}}(x-u)^{2}(x-v)^{2}+\ell_{1}(x) .
$$

The additional condition $Q_{2}^{\prime}(u)=0$ for a double zero yields

$$
d=\frac{1}{2} \frac{(w-v)(u+w)+2(w-u) w}{(w-v)+(w-u)} .
$$

The extremal support $\{u, v, w\}=\{\varphi(x, \psi(x)), \psi(x), x\}$, which by (II.9) and the involution property of $\psi(x)$ is feasible provided $x \geq \bar{c}$, yields the formula for $d(x)$ as well as the maximal stop-loss transform $\pi_{\max }^{(4)}(d)=p(w) \cdot(w-d)$.

Finally, it remains to be shown that $(\pi \circ d)(x)$ is a well-defined function. In case (1) and for the limiting case $x \rightarrow c$ the triatomic extremal support $\{x, \varphi(x$, $\psi(x)), \psi(x)\}$ converges to the biatomic support $\{c, \bar{c}\}$ and $d(x)$ converges to $\frac{1}{2} \gamma$. By symmetry, the same holds in case (2) for the limiting case $x \rightarrow \bar{c}$. Furthermore $d(x)$ is strictly increasing. Therefore, in case (1) the function $d(x)$ maps $(-\infty, c]$ one-to-one on $\left(-\infty, \frac{1}{2} \gamma\right]$, and in case (2) it maps $(\bar{c}, \infty]$ one-to-one on $\left[\frac{1}{2} \gamma, \infty\right)$. It follows that $(\pi \circ d)(x)$ is a well-defined function.

\section{REFERENCES}

ACERBI, C. (2001) Risk aversion and coherent risk measures: a spectral representation theorem. Working paper http://www.gloriamundi.org/var/wps.html.

ACERBI, C. and Tasche, D. (2001a) On the coherence of expected shortfall. Working paper http://www.gloriamundi.org/var/wps.html.

ACERBI, C. and Tasche, D. (2001b). Expected shortfall: a natural coherent alternative to valueat-risk. Working paper http://www.gloriamundi.org/var/wps.html. 
ARTZNER, P. (1999) Application of coherent risk measures to capital requirements in insurance. North American Actuarial Journal 3(2), 11-25.

ArTzNer, P., Delbaen, F., Eber, J.-M. and Heath, D. (1997) Thinking coherently. RISK 10(11), 68-71.

Artzner, P., Delbaen, F., Eber, J.-M. and Heath, D. (1999) Coherent measures of risk. Mathematical Finance 9(3), 203-28.

Bertsimas, D., Lauprete, G.J. and SAmarov, A. (2000) Shortfall as a risk measure: properties, optimization and applications. Working paper, Sloan School of Management, MIT.

Cossette, H., Denuit, M., Dhaene, J. and Marceau, E. (2001) Stochastic approximations of present value functions. Bulletin of the Swiss Association of Actuaries, 15-28.

DelBaEN, F. (2000) Coherent risk measures. Blätter der Deutschen Gesellschaft für Versicherungsmathematik XXIV(4), 733-39.

Denuit, M., Genest, C. and Marceau, E. (1999) Stochastic bounds on sums of dependent risks. Insurance: Mathematics and Economics 25, 85-104.

DE VYLDER, F. (1996) Advanced Risk Theory. A Self-Contained Introduction. Editions de l'Université de Bruxelles, Collection Actuariat.

Dhaene, J., Wang, S., Young, V. and Goovaerts, M.J. (2000) Comonotonicity and maximal stop-loss premiums. Bulletin of the Swiss Association of Actuaries, 99-113.

Durrleman, V., Nikeghbali, A. and Roncalli, T. (2000) How to get bounds for distribution convolutions? A simulation study and an application to risk management. Working paper, available from http://gro.creditlyonnais.fr/content/rd/wp.html.

Embrechrs, P. (1995) A survival kit to quantile estimation. UBS Quant Workshop, Zürich.

EMBRECHTS, P., HöING, A. and JURI, A. (2001) Using copulae to bound the value-at-risk for functions of dependents risks. Preprint ETHZ, available from http://www.math.ethz.ch/ embrechts.

Frank, M.J., Nelsen, R.B., Schweizer, B. (1987) Best-possible bounds on the distribution of a sum - a problem of Kolmogorov. Probability Theory and Related Fields 74, 199-211.

Guiard, V. (1980) Robustheit I. Probleme der angewandten Statistik, Heft 4. FZ für Tierproduktion Dummerstorf-Rostock.

Heilmann, W.-R. (1987) A premium calculation principle for large risks. In: Operations Research Proceedings 1986, 342-51.

HÜrLIMANN, W. (1994) Splitting risk and premium calculation. Bulletin of the Swiss Association of Actuaries, 229-49.

HÜRLIMANN, W. (1996) Improved analytical bounds for some risk quantities. ASTIN Bulletin 26(2), $185-99$.

HÜrlimanN, W. (1997a) Best bounds for expected financial payoffs (I) algorithmic evaluation. Journal of Computational and Applied Mathematics 82, 199-212.

HÜRLIMANN, W. (1997b) Best bounds for expected financial payoffs (II) applications. Journal of Computational and Applied Mathematics 82, 213-227.

HÜrLimanN, W. (1998a) On distribution-free safe layer-additive pricing. Insurance: Mathematics and Economics 22, 277-85.

HÜrlimanN, W. (1998b) Extremal Moment Methods and Stochastic Orders - Application in Actuarial Science. Research monograph (available from the author).

HürLimANN, W. (1999) Bounds for actuarial present values under the fractional independence age assumption (with discussions). North American Actuarial Journal 3(3), 70-84.

HÜrLimanN, W. (2000) Higher-degree stop-loss orders and right-tail risk. Manuscript (available from the author).

HÜrLIMANN, W. (2001a) Distribution-free comparison of pricing principles. Insurance: Mathematics and Economics 28, 351-360.

HÜrLIMANN, W. (2001b) Analytical evaluation of economic risk capital for portfolios of gamma risks. ASTIN Bulletin 31, 107-122.

HÜrLIMANN, W. (2001c) Financial data analysis with two symmetric distributions. First Prize in the Gunnar Benktander ASTIN Award Competition 2000. ASTIN Bulletin 31, 187-211.

HÜrLIMANN, W. (2001d) Analytical evaluation of economic risk capital and diversification using linear Spearman copulas. Working paper, available from http://www.gloriamundi.org/var/wps.html.

HürlimanN, W. (2001e) Conditional value-at-risk bounds for compound Poisson risks and a normal approximation. Working paper http://www.gloriamundi.org/var/wps.html. 
Jansen, K., Haezendonck, J. and GoovaerTs, M.J. (1986) Analytical upper bounds on stoploss premiums in case of known moments up to the fourth order. Insurance: Mathematics and Economics 5, 315-334.

KaAs, R., Heerwatrden, van A.E. and Goovaerts, M.J. (1994) Ordering of Actuarial Risks. CAIRE Education Series 1, Brussels.

KARLIN, S. and STUDDEN, W.J. (1966) Tchebycheff systems: with applications in Analysis and Statistics. Interscience Publishers, J. Wiley. New York.

Kusuoka, S. (2001) On law invariant coherent risk measures. In: Kusuoka, S. (Ed.). Advances in Mathematical Economics, 3, 83-95. Springer.

LI, D.X. (1999) Value-at-risk based on the volatility, skewness and kurtosis. Working paper http:// www.gloriamundi.org/var/wps.html.

LuCiano, E. and MARENA, M. (2001) Value at Risk bounds for portfolios of non-normal returns. Working paper http://www.gloriamundi.org/var/wps.html.

MAKAROV, G.D. (1981) Estimates for the distribution function of a sum of two random variables when the marginal distributions are fixed. Theory of Probability and its Applications 26, 803-806.

Mammana, C. (1954) Sul Problema Algebraico dei Momenti. Scuola Norma Sup. Pisa 8, 133-140.

Pearson, K. (1916) Mathematical contributions to the theory of evolution XIX; second suppl. to a memoir on skew variation. Phil. Trans. Royal Soc. London, Ser. A 216, 432.

Pflug, G. (2000) Some remarks on the value-at-risk and the conditional value-at-risk. In: Uryasev, S. (Ed.). Probabilistic Constrained Optimization: Methodology and Applications. Kluwer Academic Publishers.

RockAfEllar, R.T. and URYASEv, S. (2000) Optimization of conditional value-at-risk. The Journal of Risk 2(3), 21-41 (also available from http://www.ise.ufl.edu/uryasev).

RoCKAFELlAR, R.T. and URYASEV, S. (2001) Conditional value-at-risk for general loss distributions. Appears in Journal of Banking and Finance.

RÜSCHENDORF, L. (1982) Random variables with maximum sums. Advances in Applied Probability 14, 623-32.

Shaked, M. and Shanthikumar, J.G. (1994) Stochastic orders and their applications. Academic Press, New York.

StoyaN, D. (1977) Qualitative Eigenschaften und Abschätzungen Stochastischer Modelle. AkademieVerlag, Berlin. (English version (1983). Comparison Methods for Queues and Other Stochastic Models. J. Wiley, New York).

Testuri, C.E. and Uryasev, S. (2000) On relation between expected regret and conditional valueat-risk. Working paper http://www.ise.ufl.edu/uryasev.

WANG, S. (1998) An actuarial index of the right-tail index. North American Actuarial Journal 2(2), 88-101.

WILKINS, J.E. (1944) A note on skewness and kurtosis. Annals of Mathematical Statistics 15, 333-35.

WIRCH, J.L. (1999) Raising value at risk. North American Actuarial Journal 3(2), 106-115.

WIRCH, J.L. and HARDY, M.R. (1999) A synthesis of risk measures for capital adequacy. Insurance: Mathematics and Economics 25, 337-47.

YAMAI, Y. and YoshiBA, T. (2001a) Comparative analyses of expected shortfall and value-at-risk: their estimation error, decomposition, and optimization. Working paper http://www.gloriamundi. org/var/wps.html.

YAMAI, Y. and YoshiBA, T. (2001b) On the validity of value-at-risk: comparative analyses with expected shortfall. Working paper http://www.gloriamundi.org/var/wps.html.

YoshIBA, T. and YAMAI, Y. (2001) Comparative analyses of expected shortfall and value-at-risk (2): expected utility maximization and tail risk. Working paper http://www.gloriamundi.org/var/ wps.html.

WERNER HÜRLIMANN

Value and Risk Management

Winterthur Life and Pensions

Postfach $300-\mathrm{CH}-8401$ Winterthur - Switzerland

Tel. $+41-52-2615861$

E-mail: werner.huerlimann@winterthur.ch 